\title{
THE RING OF POLYNOMIAL FUNCTORS OF PRIME DEGREE
}

\author{
ALEXANDER ZIMMERMANN
}

\begin{abstract}
Let $\hat{\mathbb{Z}}_{p}$ be the ring of $p$-adic integers. We prove in the present paper that the category of polynomial functors from finitely generated free abelian groups to $\hat{\mathbb{Z}}_{p}$-modules of degree at most $p$ is equivalent to the category of modules over a particularly well understood ring, called Green order. This case was conjectured by Yuri Drozd.
\end{abstract}

\section{INTRODUCTION}

Polynomial functors attained a lot of interest in recent years by at least two major discoveries. First, in [19] Henn, Lannes and Schwartz showed that the category of analytic functors from the category of finite-dimensional vector spaces to to the category of vector spaces over the same field of characteristic $p$ is equivalent to the category of unstable modules over the $\bmod p$ Steenrod algebra modulo nilpotent objects. Second, Franjou, Friedlander, Scorichenko and Suslin in [15, Friedlander and Suslin in [16, Touzé in [35] as well as Touzé and van der Kallen in [36] use strict polynomial functor to prove the finite generation of cohomology of group schemes and to compute Ext-groups of modules over general linear groups. More recently Djament and Vespa studied stable homology of orthogonal, symplectic and unitary groups using some category of polynomial functors [5, 7, 37, 39]. For definitions and more ample remarks of these concepts we refer to Section 1 .

The category $\mathcal{A}_{R}$ of polynomial functors $\mathbb{Z}-$ free $\longrightarrow R-\bmod$ for a commutative ring $R$ is a classical object in algebraic topology (cf. Eilenberg, MacLane [10]). Let $\mathcal{A}_{R}^{n}$ be the full subcategory of at most degree $n$ polynomial functors in $\mathcal{A}_{R}$. Quadratic functors were characterized by Baues [2] as modules over a particular algebra. Baues, Dreckmann, Franjou and Pirashvili show in [3] that $\mathcal{A}_{R}^{n}$ is a module category of finitely generated $R$-algebra $\Gamma_{R}^{n}$ as well. This description was used by Drozd to show in [8] that $\mathcal{A}_{\widehat{\mathbb{Z}}_{2}}^{2}$ and in [9] that $\mathcal{A}_{\widehat{\mathbb{Z}}_{3}}^{3}$ are two very explicitly given classical orders over $\hat{\mathbb{Z}}_{2}$ and $\hat{\mathbb{Z}}_{3}$ respectively, whose representation theory is completely understood. In particular, each of them admit only a finite number of indecomposable lattices. Here, and in the sequel, we denote by $\hat{\mathbb{Z}}_{p}$ the ring of $p$-adic integers, and by $\mathbb{F}_{q}$ the field with $q$ elements. These orders were introduced

Received by the editors April 17, 2013 and, in revised form, July 29, 2013 and August 12, 2013.

2010 Mathematics Subject Classification. Primary 16H10; Secondary 20C30, 20J06, 55R40.

Key words and phrases. Polynomial functors, Green orders, Brauer tree algebras, Schur algebras, recollement diagram, representation type.

This research was supported by a grant "PAI alliance" from the Ministère des Affaires Étrangères de France and the British Council. The author acknowledges support from STIC Asie of the Ministère des Affaires Étrangères de France. 
by Roggenkamp in [34. Recall that an $R$-order over an integral domain $R$ is an $R$-algebra $\Lambda$, finitely generated projective as $R$-modules and so that $K \otimes_{R} \Lambda$ is a semisimple $K$-algebra, with $K$ being the field of fractions of $R$.

Drozd conjectures at the end of [9] that $\mathcal{A}_{\hat{\mathbb{Z}}_{p}}^{p}$ should be equivalent to the module category of a particular Green order $\Lambda_{p}$ over $\hat{\mathbb{Z}}_{p}$ for all primes $p$. Drozd proves the case $p=2$ and $p=3$ by explicitly associating the generators of the ring given by Baues or Baues, Dreckmann, Franjou and Pirashvili respectively to matrices in the corresponding matrix rings and computes the kernel and the image of the so-defined mapping. The relations in [3] are sufficiently involved so that going beyond $p=3$ by this method seems not to be realistic.

In this paper we prove Drozd's conjecture. Our method is conceptual. We develop a recollement diagram of $\mathcal{A}_{\mathbb{F}_{p}}^{n}$ by $\mathcal{A}_{\mathbb{F}_{p}}^{n-1}$ and the module category of the group ring $\mathbb{F}_{p} \mathfrak{S}_{n}$, analogous to the one described by Schwartz [40, Section 5.5] for functors $\mathbb{F}_{p}-\bmod \longrightarrow \mathbb{F}_{p}-\bmod$. This recollement diagram for $\mathcal{A}_{\mathbb{F}_{p}}^{n}$ may be of independent interest since it is completely general. It actually already appears in a work of Pirashvili [27, as was indicated by the referee. Comparison of these two diagrams gives much information. A second ingredient then is the study of various Ext-groups between simple functors, using work of Franjou, Friedlander, Scorichenko and Suslin [15. The third main ingredient is the explicit projective functor mapping to the reduction modulo $p$ functor. It should be noted that we do not actually use the ring defined by Baues, Dreckmann, Franjou and Pirashvili in [3. We just use that there is an algebra which is finitely generated, so that by the Krull-Schmidt property, lifting of idempotents and similar properties are valid for $\mathcal{A}_{\hat{\mathbb{Z}}_{p}}^{n}$. For this reason we do not give a Morita bimodule between the Baues, Dreckmann, Franjou and Pirashvili ring and the order we get. As an application of our result, we count the number of 'torsion free' indecomposable polynomial functors in $\mathcal{A}_{\hat{\mathbb{Z}}_{p}}^{p}$.

Our paper is organized as follows. In Section 1 we give the essential definitions and relate the different concepts. In Section 2 we recall some of the most important discoveries used in the sequel. Section 3 describes the classical recollement diagrams as well as the new one we have to use for $\mathcal{A}_{\mathbb{F}_{p}}$, and we first derive consequences. The first main result is proved in Section 5. We give the structure of $\mathcal{A}_{\mathbb{F}_{p}}^{p}$ there. Finally, in Section 6 we determine $\mathcal{A}_{\widehat{\mathbb{Z}}_{p}}^{p}$ and prove the second main theorem.

\section{Generalities on polynomial functors}

1.1. Definitions. Let $\mathcal{A}$ be a category with direct sums and $\mathcal{B}$ be a category with direct sums and kernels. Then, following Eilenberg and Mac Lane [10] define the cross effect $F^{(1)}$ of a functor $F: \mathcal{A} \longrightarrow \mathcal{B}$ to be the bifunctor $\mathcal{A} \times \mathcal{A} \longrightarrow \mathcal{B}$ defined on objects by $F^{(1)}(V \mid W):=\operatorname{ker}(F(V \oplus W) \longrightarrow F(V) \oplus F(W))$, and on morphism by the naturality of the construction. For $n \geq 1$, the $n$-th cross effect $F^{(n)}$ is the cross effect of the $n-1$-st cross effect of $F$, seen as a functor in the first variable. Hence,

$$
\begin{aligned}
F^{(n)}\left(V|W| V_{1}|\ldots| V_{n-1}\right):= & \operatorname{ker}\left(F^{(n-1)}\left(V \oplus W\left|V_{1}\right| \ldots \mid V_{n-1}\right)\right. \\
& \left.\longrightarrow F^{(n-1)}\left(V\left|V_{1}\right| \ldots \mid V_{n-1}\right) \oplus F^{(n-1)}\left(W\left|V_{1}\right| \ldots \mid V_{n-1}\right)\right)
\end{aligned}
$$


for objects $V, W, V_{1}, \ldots, V_{n-1}$ in $\mathcal{A}$. Suppose in the sequel $F(0)=0$. A functor $F$ is said to be a polynomial of degree at most $n$ if $F^{(n)}=0$ for an $n \in \mathbb{N}$ (see Pirashvili [28]). Given a commutative ring $R$, let $R-\bmod$ be the category of finitely generated $R$-modules, let $R-\operatorname{Mod}$ be the category of all $R$-modules, and let $R$-free be the category of finitely generated free $R$-modules. Further, call $\mathcal{F}_{R}^{n}$ the category of polynomial functors of degree at most $n$ from $R-\bmod$ to $R-\bmod$, and let $\mathcal{F}_{R}:=\lim _{n} \mathcal{F}_{R}^{n}$. Moreover, let $\mathcal{A}_{R}^{n}$ be the category of polynomial functors of degree at most $n$ from $\mathbb{Z}-$ free to $R-\bmod$, and let $\mathcal{A}_{R}:=\lim _{n} \mathcal{A}_{R}^{n}$. All these categories are abelian. Observe that additive functors are exactly the degree 1 polynomial functors. The only degree 0 polynomial functor is the trivial functor due to our hypothesis that $F(0)=0$.

Friedlander and Suslin define in [16, Definition 2.1] the category of strict polynomial functors $\mathcal{P}_{k}$ over a field $k$. A strict polynomial functor $F$ is defined by associating to each finite-dimensional $k$-vector space a $k$-vector space $F(V)$ and to associate for any two finite-dimensional $k$-vector spaces $V$ and $W$ an element in

$$
S^{*}\left(\operatorname{Hom}_{k}\left(\operatorname{Hom}_{k}(V, W), k\right)\right) \otimes \operatorname{Hom}_{k}(F(V), F(W))
$$

which in addition satisfy the usual compatibility relations for compositions and the identity. Each of these elements can be interpreted as mapping $H_{o m}(V, W) \longrightarrow$ $H_{\mathrm{om}}(F(V), F(W))$ by interpreting the formal polynomial

$$
S^{*}\left(\operatorname{Hom}_{k}\left(\operatorname{Hom}_{k}(V, W), k\right)\right) \otimes \operatorname{Hom}_{k}(F(V), F(W))
$$

as a polynomial mapping, so that any strict polynomial functor induces a polynomial functor $k-\bmod \longrightarrow k-\operatorname{Mod}$. Hence, we have a forgetful functor $\mathcal{P}_{k} \longrightarrow \mathcal{F}_{k}$. It is shown in [16] that the category of exact degree $n$ strict polynomial functors $\mathcal{P}_{k}^{n}$ from $k-\bmod$ to $k-\operatorname{Mod}$ for a field $k$ is equivalent to the category of modules over the Schur algebra $S_{k}(n, n)$. Moreover, $\mathcal{P}_{k}=\bigoplus_{n} \mathcal{P}_{k}^{n}$. Finally, the forgetful functor $\mathcal{P}_{k} \longrightarrow \mathcal{F}_{k}$ sends a strict polynomial functor of degree at most $d$ to a polynomial functor of degree at most $d$ (cf. [29, Remark 4.1]).

A main theme in Section 1 in particular is the question of base change. In Section 5 we shall define for any commutative rings $R$ and $S$ a particular degree $d$ polynomial functor

$$
R[-] /\left(I^{d+1}\right): S-\text { free } \rightarrow R-\operatorname{Mod}
$$

which assigns to every free $S$-module $V$ the quotient of the group ring $R[V]$ of the additive group $V$ over $R$ by the $d+1$-th power of the augmentation ideal $I$. Look at the case $S=\mathbb{Z}$. A key idea, due to Pirashvili, is to replace the morphisms $H_{\mathrm{O}}\left(\mathbb{Z}^{n}, \mathbb{Z}^{m}\right)$ in the category of free abelian groups by $R\left[\operatorname{Hom}_{\mathbb{Z}}\left(\mathbb{Z}^{n}, \mathbb{Z}^{m}\right)\right] /\left(I^{d+1}\right)$. This construction does not allow base change. Strict polynomial functors basically replace $\operatorname{Hom}_{\mathbb{Z}}\left(\mathbb{Z}^{n}, \mathbb{Z}^{m}\right)$ by $S^{d}\left(H o m_{\mathbb{Z}}\left(\mathbb{Z}^{n}, \mathbb{Z}^{m}\right)\right)$, which does admit base change. Comparison between these two constructions is the main theme in Section 1

\subsection{Functors with values in characteristic 0 .}

Lemma 1.1. Let $R$ be an integral domain of characteristic 0 . If $F$ is a polynomial functor $F: \mathbb{Z}-$ free $\longrightarrow R-\bmod$ of degree $d$, then $\operatorname{Hom}_{\mathbb{Z}}\left(\mathbb{Z}^{n}, \mathbb{Z}^{m}\right) \stackrel{F}{\longrightarrow}$ Hom $_{R}\left(F\left(\mathbb{Z}^{n}\right), F\left(\mathbb{Z}^{m}\right)\right)$ is a polynomial of degree $d$ in the $n \cdot m$ coordinate functions $\operatorname{Hom}_{\mathbb{Z}}\left(\mathbb{Z}^{n}, \mathbb{Z}^{m}\right)$. 
Proof. We shall show by induction on $n+d$ that for any $k$ homomorphisms $f_{1}, f_{2}, \ldots, f_{k} \in \operatorname{Hom}_{\mathbb{Z}}\left(\mathbb{Z}^{n}, \mathbb{Z}^{m}\right)$ and integers $\lambda_{1}, \lambda_{2}, \ldots, \lambda_{k} \in \mathbb{Z}$ one gets that $F\left(\lambda_{1} f_{1}+\lambda_{2} f_{2}+\cdots+\lambda_{k} f_{k}\right)$ is a degree $d$ polynomial in the variables $\lambda_{1}, \lambda_{2}, \ldots, \lambda_{k}$. If the degree of $F$ is 1 , there is nothing to show since then the functor is linear. Let $n>1$. Now, we know that

$$
F\left(\mathbb{Z}^{n-1} \oplus \mathbb{Z}\right)=F\left(\mathbb{Z}^{n-1}\right) \oplus F(\mathbb{Z}) \oplus F^{(1)}\left(\mathbb{Z}^{n-1} \mid \mathbb{Z}\right)
$$

Hence, the restriction $f_{i}^{\prime}$ of each of the $f_{i}$ to $\mathbb{Z}^{n-1}$ and the restriction $f_{i}^{\prime \prime}$ to the last component $\mathbb{Z}$ define morphisms $F\left(\sum_{i=1}^{k} \lambda_{i} f_{i}^{\prime}\right): F\left(\mathbb{Z}^{n-1}\right) \longrightarrow F\left(\mathbb{Z}^{m}\right)$, $F\left(\sum_{i=1}^{k} \lambda_{i} f_{i}^{\prime \prime}\right): F(\mathbb{Z}) \longrightarrow F\left(\mathbb{Z}^{m}\right)$ and $F\left(\sum_{i=1}^{k} \lambda_{i} f_{i}\right): F^{(1)}\left(\mathbb{Z}^{n-1} \mid \mathbb{Z}\right) \longrightarrow F\left(\mathbb{Z}^{m}\right)$. In the first two cases, the dimension of the source space is less than $n$, while the degree of $F$ is unchanged, whereas in the third case the dimension of the source space is $n$, but the degree of the functor is $d-1$. So, in any of these cases by the induction hypothesis we can express $F\left(\sum_{i=1}^{k} \lambda_{i} f_{i}\right)$ as a polynomial of degree $n$ in the variables $\lambda_{1}, \lambda_{2}, \ldots, \lambda_{k}$.

We are left with the case $n=1$. The very same reduction applied to the image and induction on $m+d$ implies that one can suppose that $m=1$. But then, Eilenberg and MacLane [10, (8.3)] show that for $\lambda \in \mathbb{Z}$ one has

$$
F(\lambda \cdot)=F((\lambda-1) \cdot)+F(1)+F^{(1)}(\lambda \cdot \mid 1),
$$

where $F(1)$ is the identity. Now,

$$
F(\lambda \cdot)-F((\lambda-1) \cdot)=F(1)+F^{(1)}(\lambda \cdot \mid 1),
$$

where by the induction hypothesis, since the degree of $F^{(1)}$ is less than the degree of $F$, the right hand side $F(1)+F^{(1)}(\lambda \cdot \mid 1)$ is a polynomial of degree $d-1$ in $\lambda$.

We shall now adapt an argument of Kuhn [22, Lemma 4.8] to this slightly more general situation. We claim that a function $f: \mathbb{Z} \longrightarrow R$ is a polynomial if and only if some derivative $f^{(r)}$ vanishes, where $f^{(r)}(n)=f^{(r-1)}(n)-f^{(r-1)}(n-1)$.

We assume for the moment that $R$ contains $\mathbb{Q}$. Suppose $f$ is a polynomial. Then, it is clear that $f^{(\operatorname{deg}(f)+1)}=0$. Suppose to the contrary that $f^{(r)}=0$. The polynomials $\left(\begin{array}{l}X \\ k\end{array}\right):=\frac{X \cdot(X-1) \cdots \cdot(X-k+1)}{k !}$ for $k \in\{0,1, \ldots, d\}$ form an $R$-basis of the polynomials of degree at most $d$ in $R[X]$, since $d$ ! is invertible in $R$. Moreover, $\left(\begin{array}{c}X \\ k\end{array}\right)-\left(\begin{array}{c}X-1 \\ k\end{array}\right)=\left(\begin{array}{c}X \\ k-1\end{array}\right)$. Now, by induction, $f^{(s)}$ is a polynomial, and hence a linear combination of polynomials $\left(\begin{array}{l}X \\ k\end{array}\right)$. The relation $\left(\begin{array}{c}X \\ k\end{array}\right)-\left(\begin{array}{c}X-1 \\ k\end{array}\right)=\left(\begin{array}{c}X \\ k-1\end{array}\right)$ gives a polynomial $h^{(s-1)}$ so that $\left(h^{(s-1)}\right)^{(1)}=f^{(s)}$. By induction on $n$, the values $g^{(1)}(n)=$ $g(n)-g(n-1)$ determine the values $g(n)$ up to the value of $g(0)$. Therefore, up to this constant value, $f^{(s-1)}=h^{(s-1)}$.

Now, suppose $R$ is an integral domain of characteristic 0 . Then, since $R \subseteq$ $\operatorname{frac}(R), f$ can be considered as being in values $\operatorname{frac}(R)$ which contains $\mathbb{Q}$. This proves the claim.

Now, define $f(\lambda):=F(\lambda \cdot)$ and apply the claim to conclude that $F(\lambda \cdot)$ is polynomial of degree $d$.

Let $K$ be a field. A priori the category $\mathcal{A}_{K}$ is different from the category $\mathcal{F}_{K}$. Nevertheless, in some cases we get one inclusion.

Lemma 1.2. Let $K$ be either a prime field of finite characteristic or let $K$ be a prime field of characteristic 0 . Let $\left(\circ\left(K \otimes_{\mathbb{Z}}-\right)\right)^{*}: \mathcal{F}_{K} \longrightarrow \mathcal{A}_{K}$ be the functor 
defined by $\left(\circ\left(K \otimes_{\mathbb{Z}}-\right)\right)^{*}(F):=F \circ\left(K \otimes_{\mathbb{Z}}-\right)$. Then, $\left(\circ\left(K \otimes_{\mathbb{Z}}-\right)\right)^{*}$ induces a fully faithful embedding $\mathcal{F}_{K} \hookrightarrow \mathcal{A}_{K}$.

Proof. The functor $\circ\left(-\otimes_{\mathbb{Z}} K\right): \mathcal{F}_{K} \longrightarrow \mathcal{A}_{K}$ induces for any two functors $F$ and $G$ in $\mathcal{F}_{K}$ a mapping

$$
\varphi: \operatorname{Hom}_{\mathcal{F}_{K}}(F, G) \longrightarrow \operatorname{Hom}_{\mathcal{A}_{K}}\left(F \circ\left(-\otimes_{\mathbb{Z}} K\right), G \circ\left(-\otimes_{\mathbb{Z}} K\right)\right) .
$$

We shall need to show that this mapping is an isomorphism.

Injectivity: Let $\eta_{1}$ and $\eta_{2}$ be two objects in $\operatorname{Hom}_{\mathcal{F}_{K}}(F, G)$. Suppose $\varphi\left(\eta_{1}\right)=$ $\varphi\left(\eta_{2}\right)$. Observe that for any $V \in \mathbb{Z}-$ free we have

$$
\left(\varphi\left(\eta_{1}\right)\right)(V)=\eta_{1}\left(K \otimes_{\mathbb{Z}} V\right) \in \operatorname{Hom}_{K}\left(F\left(K \otimes_{\mathbb{Z}} V\right), G\left(K \otimes_{\mathbb{Z}} V\right)\right),
$$

and likewise for $\eta_{2}$, satisfying that for any $V$ and $W$ and any $\rho \in H o m_{\mathbb{Z}}(V, W)$ one has $\left(\eta_{1}\left(K \otimes_{\mathbb{Z}} V\right)\right) \circ \hat{G}(\rho)=\hat{F}(\rho) \circ\left(\eta_{1}\left(K \otimes_{\mathbb{Z}} W\right)\right)$.

Since $\circ\left(-\otimes_{\mathbb{Z}} K\right)$ is dense, $\eta_{1}$ and $\eta_{2}$ coincide on every object of $K-\bmod$, and so $\eta_{1}=\eta_{2}$.

As a consequence, without any further hypothesis,

$$
\varphi: \operatorname{Hom}_{\mathcal{F}_{K}}(F, G) \hookrightarrow \operatorname{Hom}_{\mathcal{A}_{K}}\left(F \circ\left(-\otimes_{\mathbb{Z}} K\right), G \circ\left(-\otimes_{\mathbb{Z}} K\right)\right) .
$$

Surjectivity: Let $\eta \in H_{o m_{\mathcal{A}_{K}}}\left(F \circ\left(-\otimes_{\mathbb{Z}} K\right), G \circ\left(-\otimes_{\mathbb{Z}} K\right)\right)$ be a natural transformation. We need to show that there is a natural transformation $\eta^{\prime} \in \operatorname{Hom}_{\mathcal{F}_{K}}(F, G)$ so that $\varphi\left(\eta^{\prime}\right)=\eta$.

In the case when $K$ is a prime field of finite characteristic, define for any $V \in$ $K-\bmod$ the mapping $\eta^{\prime}(V):=\eta\left(P_{V}\right)$, where $P_{V}$ is a fixed chosen projective cover of $V$, so that $K \otimes_{\mathbb{Z}} P_{V}=V$.

In the case when $K$ is of characteristic 0 , fix for any $K$-vector space $V$ a free abelian subgroup $P_{V}$ so that $K \otimes_{\mathbb{Z}} P_{V}=V$. Define $\eta^{\prime}(V):=\eta\left(P_{V}\right)$.

We need to show that $\eta^{\prime}$ is a natural transformation.

Let $\varphi \in \operatorname{Hom}_{K}(V, W)$.

Consider first the case of $K$ being a prime field of finite characteristic. Since $P_{V}$ and $P_{W}$ are projective covers of $V$ and $W$ as abelian groups, there is a $\hat{\varphi} \in$ $\operatorname{Hom}_{\mathbb{Z}}\left(P_{V}, P_{W}\right)$ so that $K \otimes_{\mathbb{Z}} \hat{\varphi}=\varphi$ under the identification $K \otimes_{\mathbb{Z}} P_{V}=V$ and $K \otimes_{\mathbb{Z}} P_{W}=W$. Since $\eta$ is a natural transformation, $\hat{G}(\hat{\varphi}) \circ \eta\left(P_{V}\right)=\eta\left(P_{W}\right) \circ \hat{F}(\hat{\varphi})$. But, by definition, $\hat{G}(\hat{\varphi})=G(\varphi)$ and $\hat{F}(\hat{\varphi})=F(\varphi)$, as well as $\eta\left(P_{W}\right)=\eta^{\prime}(W)$ and $\eta\left(P_{V}\right)=\eta^{\prime}(V)$. So, $\eta^{\prime}$ is a natural transformation.

Suppose now that $K$ is a prime field of characteristic 0 . Since $\mathcal{F}_{K}=\mathcal{P}_{K}$ in this case, we know that $F$ (and $G$ resp.) are polynomial laws transforming any linear mapping $V \longrightarrow W$ into a linear mapping $F(V) \longrightarrow F(W)$ (and $G(V) \longrightarrow G(W)$ resp.) which depends polynomially on the coefficients of any matrix representation with respect to any fixed bases. We know that for any $\mathbb{Z}$-linear mapping $\hat{\varphi}: P_{V} \longrightarrow$ $P_{W}$

(‡) $G\left(K \otimes_{\mathbb{Z}} \varphi\right) \circ \eta\left(P_{V}\right)=\hat{G}(\hat{\varphi}) \circ \eta\left(P_{V}\right)=\eta\left(P_{W}\right) \circ \hat{F}(\hat{\varphi})=\eta\left(P_{W}\right) \circ F\left(K \otimes_{\mathbb{Z}} \varphi\right)$.

Since this equation holds when evaluated on an infinite set of coefficients, since $\mathbb{Z}$ and $K$ are both infinite, the above equation $(\ddagger)$ holds as a polynomial equation.

Therefore, the equation holds as well for $\varphi$, since there the only difference is that the polynomials are evaluated not only on integer coefficients, but also on coefficients in $K$. Since the equation holds as polynomials, this equation also holds true evaluated on $K$. 
Therefore again $\eta_{W}^{\prime} \circ F(\varphi)=G(\varphi) \circ \eta_{V}^{\prime}$. This proves that $\eta^{\prime}$ is a natural transformation.

We are now concerned with the question when a polynomial functor $\mathbb{Z}-$ free $\longrightarrow$ $R-\bmod$ can be extended to a strict polynomial functor $R-\bmod \longrightarrow R-\bmod$ by composing with the 'extending scalars' functor $\mathbb{Z}-$ free $\stackrel{R \otimes_{\mathbb{Z}}-}{\longrightarrow} R-\bmod$. In other words we study the question when $\left(\circ\left(K \otimes_{\mathbb{Z}}-\right)\right)^{*}$ is an equivalence $\mathcal{A}_{R} \simeq \mathcal{P}_{R}$. In order to prove this, by Lemma 1.2 . one needs to show that $\left(\circ\left(K \otimes_{\mathbb{Z}}-\right)\right)^{*}$ is dense as well.

We have to deal with mainly two cases: the case of $R$ being a field of characteristic 0 and the case of $R$ being a field of characteristic $p$. We shall see that fields with characteristic 0 behave more like characteristic $\infty$. The remarks at the beginning of this section on base change properties are particularly visible in the proof of the following lemma.

Lemma 1.3. Let $R$ be a field of characteristic 0 and let $F: \mathbb{Z}-$ free $\longrightarrow R-\bmod$ be a polynomial functor of degree $d$. Then, $F$ extends to a strict polynomial functor $\hat{F}: R-$ free $\longrightarrow R-\bmod$ so that $\hat{F} \circ\left(R \otimes_{\mathbb{Z}}-\right)=F$. In particular, $\left(\circ\left(R \otimes_{\mathbb{Z}}-\right)\right)$ * induces an equivalence $\mathcal{A}_{R}^{d} \simeq \mathcal{P}_{\bar{R}}^{\leq d}$.

Proof. By Lemma 1.2 we know that $\mathcal{F}_{K}^{d} \hookrightarrow \mathcal{A}_{K}^{d}$.

We have to show that this embedding is dense. Let $F$ be a degree $d$ polynomial functor in $\mathcal{A}_{K}^{d}$. By Lemma 1.1 we know that for any $n$ and $m$ the functor $F$ induces a degree $d$ polynomial mapping with coefficients in $K$ in the coordinate functions of matrices in $H_{0} m_{\mathbb{Z}}\left(\mathbb{Z}^{n}, \mathbb{Z}^{m}\right)$. Moreover, $F$ is a functor, that is, $F(\alpha \beta)=F(\alpha) F(\beta)$ and $F\left(i d_{A}\right)=i d_{A}$ for any free abelian group $A$ and any two composable morphisms of abelian groups $\alpha$ and $\beta$.

Let $\alpha: \mathbb{Z}^{n} \longrightarrow \mathbb{Z}^{m}$ and $\beta: \mathbb{Z}^{k} \longrightarrow \mathbb{Z}^{n}$. The equation $F(\alpha \beta)=F(\alpha) F(\beta)$ translates into an equation between the evaluation of the corresponding polynomials in each degree. Since $\mathbb{Z}$ and $K$ are of characteristic 0 , the polynomial equation holds if evaluated on infinitely many values, and so the polynomial equations actually holds as polynomials. Friedlander and Suslin remark in [16, remark after Definition 2.1 that this is actually equivalent to saying that $F$ is actually a strict polynomial functor $\hat{G} \in \mathcal{P}_{K}$ of degree $d$. This proves the lemma.

\subsection{Functors with values in fields of finite characteristic.}

Lemma 1.4. Let $\mathbb{F}$ be a field of characteristic $p$ and let $F: \mathbb{Z}-$ free $\longrightarrow \mathbb{F}-\bmod$ be a polynomial functor of degree at most $p-1$ which preserves the initial object, i.e. $F(0)=0$. Then, for any homomorphism $\alpha: \mathbb{Z}^{n} \longrightarrow \mathbb{Z}^{m}$ one gets $F(p \cdot \alpha)=0$.

Proof. Let $M=\mathbb{Z}^{n}$ and $N=\mathbb{Z}^{m}$. We write $p \cdot \alpha$ in the diagram

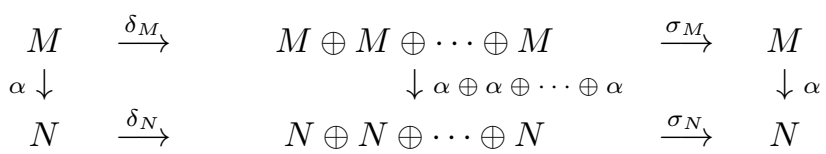

where $\delta$ is the diagonal mapping and $\sigma$ is the summation mapping, on $M$ or on $N$ respectively. 
Denote by $F^{(i)}$ the $i$-th cross effect of the functor $F$. Since $F$ is polynomial of degree at most $p$,

$$
F\left(A_{1} \oplus A_{2} \oplus \cdots \oplus A_{p}\right)=\bigoplus_{i=1}^{p} \bigoplus_{j_{1}<\cdots<j_{i}} F^{(i-1)}\left(A_{j_{1}}|\ldots| A_{j_{i}}\right)
$$

for $p$ abelian groups $A_{1}, \ldots, A_{p}$, and this decomposition is functorial with respect to these groups. Moreover, since $\operatorname{deg}(F)=p-1$ and since $F(0)=0$, one gets $F^{(p-1)}=0$.

Since $F(p \cdot \alpha)=F\left(\alpha \circ \sigma_{M} \circ \delta_{M}\right)=F(\alpha) \circ F\left(\sigma_{M} \circ \delta_{M}\right)$, it is necessary and sufficient to show that $F\left(\sigma_{M} \circ \delta_{M}\right)=0$. Now,

$$
F(M \oplus M \oplus \cdots \oplus M)=\bigoplus_{i=1}^{p} \bigoplus_{j_{1}<\cdots<j_{i}} F^{(i-1)}\left(M_{j_{1}}|\ldots| M_{j_{i}}\right),
$$

where $M_{l}=M$ is the copy of $M$ in the $l$-th position of $\bigoplus_{i=1}^{p} M$, for all $l \leq p$. The mapping $F\left(\sigma_{M} \circ \delta_{M}\right)=F\left(\sigma_{M}\right) \circ F\left(\delta_{M}\right)$ factors as a sum $\sum_{i=1}^{p} F\left(\sigma_{M}\right) \circ$ $\iota_{i} \circ \pi_{i} \circ F\left(\delta_{M}\right)$, where $\iota_{i}$ is the embedding of $\bigoplus_{j_{1}<\cdots<j_{i}} F^{(i-1)}\left(M_{j_{1}}|\ldots| M_{j_{i}}\right)$ into $F(M \oplus M \oplus \cdots \oplus M)$ and $\pi_{i}$ is the projection of $F(M \oplus M \oplus \cdots \oplus M)$ to this direct factor. But now, for $i<p,\left.F\left(\sigma_{M}\right)\right|_{\left(\oplus_{j_{1}<\cdots<j_{i}} F^{(i-1)}\left(M_{j_{1}}|\ldots| M_{j_{i}}\right)\right)} \circ \pi_{i} \circ F\left(\delta_{M}\right)$ is a sum of $p$ identical mappings, which sum up to 0 in characteristic $p$. Hence, $F\left(\sigma_{M} \circ \delta_{M}\right)=F\left(\sigma_{M}\right) \circ F^{(p-1)}(\alpha|\alpha| \ldots \mid \alpha) \circ F\left(\delta_{M}\right)=0$ using that $F^{(p-1)}=0$.

Corollary 1.5. Let $\mathbb{F}$ be a field of characteristic $p$ and let $F: \mathbb{Z}-$ free $\longrightarrow \mathbb{F}-$ mod be a polynomial functor of degree at most $p-1$ which preserves the initial object, i.e. $F(0)=0$. Then, for any two homomorphisms $\alpha: \mathbb{Z}^{n} \longrightarrow \mathbb{Z}^{m}$ and $\beta: \mathbb{Z}^{n} \longrightarrow \mathbb{Z}^{m}$ so that $\alpha-\beta \in p \cdot \operatorname{Hom}_{\mathbb{Z}}\left(\mathbb{Z}^{n}, \mathbb{Z}^{m}\right)$, one gets $F(\alpha)=F(\beta)$.

Proof. This is a consequence of the previous lemma and [10, p. 76, formula 8.5] and [10, Theorem 9.3]. Indeed, $F\left(\sum_{n=1}^{p+1} \rho_{n}\right)=\sum_{n=1}^{p+1} \sum_{i_{1}<\cdots<i_{n}} F^{(n-1)}\left(\rho_{i_{1}}|\ldots| \rho_{i_{n}}\right)$ implies

$F(\alpha+p \cdot \gamma)=F(\alpha)+F^{(p-1)}(\underbrace{\gamma|\gamma| \ldots \mid \gamma}_{p \text { factors }})+F^{(p)}(\alpha \mid \underbrace{\gamma|\gamma| \ldots \mid \gamma}_{p \text { factors }})=F(\alpha)+F(p \gamma)=F(\alpha)$

by Lemma 1.4

Lemma 1.6. Let $\mathbb{F}$ be the prime field of characteristic $p$ and let $F: \mathbb{Z}-$ free $\longrightarrow$ $\mathbb{F}-\bmod$ be a polynomial functor of degree less than or equal to $p-1$. Then, $F$ factors through the functor $\mathbb{F} \otimes_{\mathbb{Z}}-: \mathbb{Z}-$ free $\longrightarrow \mathbb{F}-$ mod. Moreover, if $F=F^{\prime} \circ\left(\mathbb{F} \otimes_{\mathbb{Z}}-\right)$, then $F$ is a polynomial of degree $m$ if and only if $F^{\prime}$ is a polynomial of degree $m$. Hence, $\left(\circ\left(\mathbb{F} \otimes_{\mathbb{Z}}-\right)\right)^{*}$ induces an equivalence $\mathcal{A}_{\mathbb{F}}^{\leq p-1} \simeq \mathcal{F}_{\mathbb{F}}^{\leq p-1}$.

Proof. Since $\left(\mathbb{F} \otimes_{\mathbb{Z}}-\right)^{*}: \mathcal{F}_{\mathbb{F}} \hookrightarrow \mathcal{A}_{\mathbb{F}}$ is a fully faithful embedding, we need to show that $\left(\mathbb{F} \otimes_{\mathbb{Z}}-\right)^{*}$ is dense. So, given a functor $F: \mathbb{Z}-$ free $\longrightarrow \mathbb{F}-\bmod$, one has to show that there is a functor $\hat{F}: \mathbb{F}-\bmod \longrightarrow \mathbb{F}-\bmod$ with $\hat{F} \circ\left(\mathbb{F} \otimes_{\mathbb{Z}}-\right)=F$.

For any $V \in \mathbb{F}-\bmod$ choose $P_{V}$ a projective cover as an abelian group. Then, $P_{V}$ is in $\mathbb{Z}$-free. By the universal property of projective covers one has for any $\alpha \in H o m_{\mathbb{F}}(V, W)$ a (non-unique) $\hat{\alpha} \in \operatorname{Hom}_{\mathbb{Z}}\left(P_{V}, P_{W}\right)$ so that

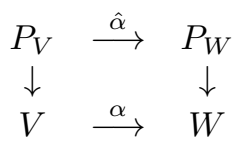


is commutative. Put

$$
\hat{F}(V):=F\left(P_{V}\right) \text { and } \hat{F}(\alpha):=F(\hat{\alpha}) .
$$

We need to show that this gives a functor $\hat{F}: \mathbb{F}-\bmod \longrightarrow \mathbb{F}-\bmod$. Let $\hat{\alpha}$ and $\hat{\alpha}^{\prime}$ be two different lifts of $\alpha: V \longrightarrow W$; then $\hat{\alpha}-\hat{\alpha}^{\prime}$ lifts the 0-mapping, and so $\hat{\alpha}-\hat{\alpha}^{\prime} \in p \cdot \operatorname{Hom}_{\mathbb{Z}}\left(P_{V}, P_{W}\right)$. Corollary 1.5 implies that $\hat{F}(\hat{\alpha})=\hat{F}\left(\hat{\alpha}^{\prime}\right)$. Using Corollary 1.5] again one gets that $\hat{F}\left(i d_{V}\right)=i d_{\hat{F}(V)}$ since $i d_{P_{V}}$ is a lift of $i d_{V}$. Moreover, let $\alpha: U \longrightarrow V$ and $\beta: V \longrightarrow W$; then choosing lifts $\hat{\alpha}: P_{U} \longrightarrow P_{V}$ and $\beta: P_{V} \longrightarrow P_{W}$, one gets that $\widehat{\alpha \beta}-\hat{\alpha} \hat{\beta}$ lifts the 0-mapping. So, $\widehat{\alpha \beta}-\hat{\alpha} \hat{\beta} \in$ $p \cdot \operatorname{Hom}_{\mathbb{Z}}\left(P_{U}, P_{W}\right)$, and again by Corollary 1.5 one has $F(\widehat{\alpha \beta})=F(\hat{\alpha} \hat{\beta})$.

\section{A REVIEW ON POLYNOMIAL FUNCTORS AND FUNCTOR COHOMOLOGY}

2.1. Polynomial functors are modules. Let $R$ be a commutative ring. We know that by a result of Baues, Dreckmann, Franjou and Pirashvili [3] a polynomial functor of degree at most $n$ from free abelian groups to $R$-modules is defined by giving $R$-modules $F_{m}(\mathbb{Z}|\mathbb{Z}| \ldots \mid \mathbb{Z})$ for all $m \leq n$ and mappings

$$
h_{k}^{m}: F_{m}(\mathbb{Z}|\mathbb{Z}| \ldots \mid \mathbb{Z}) \longrightarrow F_{m+1}(\mathbb{Z}|\mathbb{Z}| \ldots \mid \mathbb{Z})
$$

for $k \leq m \leq n-1$ and

$$
p_{k}^{m+1}: F_{m+1}(\mathbb{Z}|\mathbb{Z}| \ldots \mid \mathbb{Z}) \longrightarrow F_{m}(\mathbb{Z}|\mathbb{Z}| \ldots \mid \mathbb{Z})
$$

for $k \leq m \leq n-1$ satisfying the relations

$$
(*) h_{j}^{m} p_{i}^{m}= \begin{cases}p_{i}^{m+1} h_{j+1}^{m+1} & \text { for } j<i, \\ p_{i+1}^{m+1} h_{j}^{m+1} & \text { for } j>i, \\ 1+t_{i}^{m}+p_{i}^{m+1} h_{i+1}^{m+1}+p_{i+1}^{m+1} h_{i}^{m+1}+p_{i+1}^{m+1} t_{i}^{m+1} h_{i+1}^{m+1} & \\ +p_{i}^{m+1} t_{i+1}^{m+1} h_{i}^{m+1}+p_{i+1}^{m+1} p_{i}^{m+2} t_{i+1}^{m+2} h_{i}^{m+2} h_{i+1}^{m+1} & \text { for } j=i,\end{cases}
$$

and relations which encode functoriality of $h_{k}^{m}, p_{k}^{m}$ and of $t_{k}^{m}$.

Define the algebra $\Gamma_{R}^{n}$ over $R$ by a quiver with $n$ vertices $F_{m}(\mathbb{Z}|\mathbb{Z}| \ldots \mid \mathbb{Z})$ for any $m$ with $1 \leq m \leq n$ and arrows $h_{k}^{m}: F_{m}(\mathbb{Z}|\mathbb{Z}| \ldots \mid \mathbb{Z}) \longrightarrow F_{m+1}(\mathbb{Z}|\mathbb{Z}| \ldots \mid \mathbb{Z})$ and $p_{k}^{m+1}: F_{m+1}(\mathbb{Z}|\mathbb{Z}| \ldots \mid \mathbb{Z}) \longrightarrow F_{m}(\mathbb{Z}|\mathbb{Z}| \ldots \mid \mathbb{Z})$ subject to the relations $(*)$. Observe that the relations above do not form a set of admissible relations. The third relation though should be read as the defining equation for the symbols $t_{i}^{n}$, and this way the relations $(*)$ is a set of admissible relations. The result [3] of Baues et al. implies that $\Gamma_{R}^{n}-\bmod$ is equivalent to the category of polynomial functors of degree at most $n$.

Since the ring homomorphism $\hat{Z}_{p} \longrightarrow \mathbb{F}_{p}$ induces an embedding $\mathbb{F}_{p}-\bmod \longrightarrow$ $\hat{\mathbb{Z}}_{p}-\bmod$, we get an induced embedding $\Gamma_{\mathbb{F}_{p}}^{n}-\bmod \longrightarrow \Gamma_{\hat{\mathbb{Z}}_{p}}^{n}-\bmod$ which is also induced by the surjective ring homomorphism $\Gamma_{\hat{\mathbb{Z}}_{p}}^{n} \longrightarrow \Gamma_{\mathbb{F}_{p}}^{n}$.

Remark 2.1. We should mention that the description of [3] was recently generalised by Hartl, Pirashvili and Vespa [18] to show an isomorphism of the category of functors FreeGroups $\longrightarrow \mathbb{Z}-$ Mod with the category of what they call pseudoMackey functors. 
2.2. Some facts on functor cohomology. We shall give some facts that we will need from Franjou, Friedlander, Scorichenko and Suslin [15. Basically, these results reduce the computation of extension groups between polynomial functors to questions between extension groups between strict polynomial functors.

Let $k$ be a commutative ring. Let $\mathcal{F}(k)$ be the category of polynomial functors $k-\bmod \longrightarrow k-\bmod$ and let $\mathcal{P}(k)$ the category of strict polynomial functors between $k$-modules. Let $\mathcal{F}^{n}=\mathcal{F}^{n}(k)$ be the category of degree $n$ polynomial functors from $k-\bmod$ to $k-\bmod$. If $k=\mathbb{F}_{q}$ for $q=p^{s}$, the field with $q$ elements, we write $\mathcal{F}^{n}(k)=\mathcal{F}^{n}(q)$. In this case, for any strict polynomial functor $P$ in $\mathcal{P}(k)$ let $P^{(m)}$ be the functor twisted by the Frobenius endomorphism defined by $\mathbb{F}_{q} \ni x \mapsto x^{p^{m}} \in \mathbb{F}_{q}$.

Theorem 2.2 ([15]). Given any two homogeneous strict polynomial functors $P$ and $Q$ between $\mathbb{F}_{q}$-vector spaces for $q=p^{s}$, if the degrees of $P$ and $Q$ are different and strictly smaller than $q$, then $\operatorname{Ext}_{\mathcal{F}(k)}^{*}(P, Q)=0$. Moreover, if the degrees of $P$ and $Q$ coincide, then

$$
\lim _{m} \operatorname{Ext}_{\mathcal{P}(k)}^{*}\left(P^{(m)}, Q^{(m)}\right) \simeq \operatorname{Ext}_{\mathcal{F}(k)}^{*}(P, Q) .
$$

Frobenius twisting decreases the 'degree of homological triviality' as is shown in a result of $\mathrm{H}$. H. Andersen.

Proposition 2.3 (H. H. Andersen; see [15, Corollary 1.3]). For two homogeneous strict polynomial functors $P$ and $Q$ between $\mathbb{F}_{q}$-vector spaces of the same degree, for $q=p^{s}$ and for $m \in \mathbb{N} \cup\{0\}$ we get

$$
\operatorname{Ext}_{\mathcal{P}(k)}^{*}\left(P^{(m)}, Q^{(m)}\right) \leq \operatorname{Ext}_{\mathcal{P}(k)}^{*}\left(P^{(m+1)}, Q^{(m+1)}\right) .
$$

The first of the two statements in Theorem 2.2 actually is due to Kuhn:

Lemma $2.4([22])$. Any functor $F \in \mathcal{F}(q)$ decomposes into a direct sum $F=$ $\bigoplus_{i=0}^{q-1} F_{i}$, where $F_{i}(V):=\left\{x \in F(V) \mid F(\lambda \cdot)(x)=\lambda^{i} \cdot x \quad \forall \lambda \in \mathbb{F}_{q}\right\}$. This induces a decomposition of the category of functors between $\mathbb{F}_{q}$-vector spaces $\mathcal{F}(q)=$ $\prod_{i=0}^{q-1} \mathcal{F}(q)_{i}$.

Finally, a result due to Kuhn will be essential in the sequel.

Theorem 2.5 (N. Kuhn [23,24]). The injective envelope $I_{\mathbb{F}_{p}}$ of the trivial module in the category of analytic functors $\mathcal{F}^{\omega}\left(\mathbb{F}_{p}\right)$ from finite-dimensional $\mathbb{F}_{p}$-vector spaces to $\mathbb{F}_{p}$-vector spaces is uniserial, and the only composition factors in $\mathcal{F}^{p}\left(\mathbb{F}_{p}\right)$ are the two composition factors of $\left.\operatorname{soc}_{2}\left(I_{\mathbb{F}_{p}}\right)\right)$, where, as usual, soc 2 denotes the second layer in the socle series [4, Definition 1.2.1].

The next result of Franjou, Lannes and Schwartz implies that the categories $\mathcal{A}_{\mathbb{F}_{p}}^{p}$ and $\mathcal{F}_{\mathbb{F}_{p}}^{p}$ are different.

Theorem 2.6 (Franjou-Lannes-Schwartz [12]; Franjou-Pirashvili [13]).

$$
\operatorname{Ext}_{\mathcal{A}_{\mathbb{F}_{p}}^{p}}\left(i d, \mathbb{F}_{p} \otimes i d\right) \simeq \mathbb{F}_{p}\left[e_{1}, e_{2}, \ldots\right] /\left(e_{h}^{p} ; h \geq 0\right) \otimes \Lambda\left(\xi_{1}\right)
$$

where $\Lambda\left(\xi_{1}\right)$ is the exterior algebra in one variable with generator in degree $2 p-1$ and $e_{h}$ are generators in degree $2 p^{h}$. Moreover,

$$
\operatorname{Ext}_{\mathcal{F}_{\mathbb{F}_{p}}^{p}}(i d, i d) \simeq \mathbb{F}_{p}\left[e_{0}, e_{1}, \ldots\right] /\left(e_{h}^{p} ; h \geq 0\right) .
$$


Remark that $\mathbb{F}_{p} \otimes_{\mathbb{Z}} i d=i d$ as functors on the category $\mathbb{F}_{p}-\bmod$. As a consequence, $\operatorname{Ext}_{\mathcal{F}_{\mathbb{F}_{p}}^{p}}^{2}(i d, i d) \neq 0$. Indeed, by [12, 7.3] the following four term sequence is a non-zero element:

$$
0 \longrightarrow i d \longrightarrow S_{p} \longrightarrow S^{p} \longrightarrow i d \longrightarrow 0,
$$

where $S_{p}$ is the degree $p$ homogeneous part of the coinvariants under the $\mathfrak{S}_{p}$ action on the tensor algebra, and where $S^{p}$ is the degree $p$ homogeneous part of the invariants of the tensor algebra.

\section{ON RECOLLEMENT DIAGRAMS}

We remind the reader of the notion of a recollement diagram. A recollement diagram is given by three categories $\mathcal{A}, \mathcal{B}$ and $\mathcal{C}$ with functors

$$
\mathcal{A} \underset{\stackrel{i}{\leftarrow}}{\stackrel{q}{\stackrel{q}{\leftarrow}}} \stackrel{\stackrel{l}{\leftarrow}}{\stackrel{e}{\rightarrow}} \mathcal{C}
$$

so that

(1) $(l, e)$ and $(e, r)$ are adjoint pairs.

(2) $(q, i)$ and $(i, p)$ are adjoint pairs.

(3) $i$ is a full embedding and $e\left(B^{\prime}\right)=0 \Leftrightarrow B^{\prime} \simeq i\left(A^{\prime}\right)$ for an $A^{\prime} \in \mathcal{A}$.

(4) The adjointness morphisms $e \circ r \longrightarrow i d_{\mathcal{C}}$ and $i d_{\mathcal{C}} \longrightarrow e \circ l$ are isomorphisms.

We denote a recollement diagram as above by $(\mathcal{A}, \mathcal{B}, \mathcal{C},(e, l, r),(i, q, p))$.

We shall give a result which is a special case of a recent result of Chrysostomos Psaroudakis and Jorge Vitoria 32. We shall give our original proof below, since in our special case the proof is much easier than the proof for the general statement from 32 .

Proposition 3.1. Let $(\mathcal{A}, \mathcal{B}, \mathcal{C},(e, l, r),(i, q, p))$ be a recollement diagram. Suppose $\mathcal{B} \simeq B-\bmod$ and $\mathcal{C}=C-\bmod$ are module categories and that $\mathcal{B}$ satisfies the KrullSchmidt theorem on projective modules. Suppose that $e$ is representable. Then, $\mathcal{A}=A-\bmod$ again is a module category, and there is an idempotent $e^{\prime}$ in $B$ so that $C$ is Morita equivalent to $e^{\prime} B e^{\prime}$, and $A$ is Morita equivalent to $B / B e^{\prime} B$.

Proof. Since $e$ has a left and a right adjoint, $e$ is exact. Therefore, $e=\operatorname{Hom}_{\mathcal{B}}(P,-)$, where $P$ is a projective object. Since $l$ and $r$ are left and right adjoints to $e$,

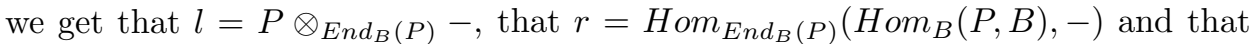
$C \simeq \operatorname{End}_{B}(P)$. Since $\mathcal{B}$ is a Krull-Schmidt category, then up to Morita equivalence, one can choose $P=B e^{\prime}$ for an idempotent $e^{\prime 2}=e^{\prime} \in B$, and we get that $C$ is Morita equivalent to $e^{\prime} B e^{\prime}$.

The third condition in the definition of a recollement diagram implies that $\mathcal{A}$ can be identified with those $B$-modules $M$ for which $e^{\prime} M=0$. Hence,

$$
\mathcal{A} \simeq\left\{M \in B-\bmod \mid e^{\prime} M=0\right\} \simeq B / B e^{\prime} B-\bmod .
$$

This proves the proposition.

Another important observation is that, by the adjointness properties, $l$ maps the projective object in $\mathcal{C}$ to projective objects in $\mathcal{B}$, and that $r$ maps the injective objects in $\mathcal{C}$ to injective objects in $\mathcal{B}$. 
3.1. Analyzing Schwartz' recollement for polynomial functors. Let $q=p^{s}$ for a prime $p$. Then, using the notation of Section 2.2 following Kuhn 25, Theorem 1.3] or Schwartz [40, §5.5] we have a recollement diagram

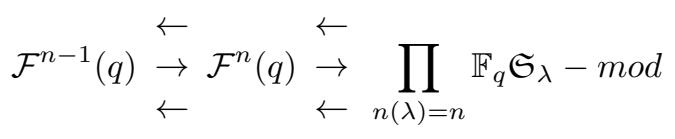

where $\mathfrak{S}_{\lambda}=\mathfrak{S}_{\lambda_{1}} \times \cdots \times \mathfrak{S}_{\lambda_{s-1}}$, where $\mathfrak{S}_{k}$ is the symmetric group on $k$ elements and where $n(\lambda):=\lambda_{0}+\cdots+\lambda_{s-1}$. Moreover, the functor $\mathcal{F}^{n}(q) \longrightarrow \prod_{n(\lambda)=n} \mathbb{F}_{q} \mathfrak{S}_{\lambda}-$ mod is representable by $i d^{\lambda}$, and for a partition $\lambda=\left(\lambda_{0} \geq \cdots \geq \lambda_{s-1}\right)$, we set $i d^{\lambda}:=\bigoplus_{j=1}^{s-1} i d^{\otimes \lambda_{j}}$.

Remark 3.2. Hence, in case $s=1$ and $n<p$, the recollement becomes

$$
\begin{aligned}
\mathcal{F}^{n-1}(p) & \rightarrow \mathcal{F}^{n}(p) \\
& \rightarrow \prod\left(\mathbb{F}_{p}-\text { mod }\right)
\end{aligned}
$$

since $\mathbb{F}_{p} \mathfrak{S}_{n}$ is semisimple, since $\mathbb{F}_{p}$ is a splitting field, and therefore its module category is equivalent to a direct product of copies $\mathbb{F}_{p}-\bmod$.

In the case $s=1$ and $n=p$, the recollement becomes

$$
\begin{aligned}
\mathcal{F}^{p-1}(p) & \leftarrow \mathcal{F}^{p}(p) \\
& \leftarrow \mathbb{F}_{p} \mathfrak{S}_{p}-\bmod .
\end{aligned}
$$

We have an immediate consequence of the above result.

Lemma 3.3. For any simple polynomial functor $F$ there is a strict polynomial functor $\hat{F}$ such that the forgetful functor, which assigns to every strict polynomial functor its polynomial functor by evaluating the polynomial as a mapping, maps $\hat{F}$ to $F$.

Proof. This is done by induction on the degree. The simple objects in $\mathcal{F}^{d}(q)$ are in bijection with the union of the simple objects in $\mathcal{F}^{d-1}(q)$ and the simple objects in $\mathbb{F}_{q} \mathfrak{S}_{d}-\bmod$. Now, any simple $\mathbb{F}_{q} \mathfrak{S}_{d}$-module is an image of a simple module of the Schur algebra $S_{\mathbb{F}_{q}}(d, d)$ under the Schur functor as is a classical fact. Since the category of degree $d$ strict polynomial functors is equivalent to the category of modules over the Schur algebra $S(d, d)$, the simple objects in $\mathcal{F}^{d}(q)$ of degree $d$ are images of a strict polynomial functor. By induction, the simple objects of degree less than $d$ are images of strict polynomial functors.

3.2. Recollement for $\mathcal{A}_{\mathbb{F}_{p}}$. For the category $\mathcal{A}_{\mathbb{F}_{p}}$ we get a similar recollement diagram.

Remark 3.4. The treatment we give for Section 3.2 was suggested by the referee, and follows paths which are not easily documented. They appeared in the special case of degree 2 in introductory remarks in 11,12. An alternative proof can be obtained, and actually this was our initial approach, using methods in the first chapter of Piriou's thesis 30,31 . We are very grateful to the referee for this useful hint.

The recollement diagram was proved in a more general situation in the meantime by Djament and Vespa [7, Theorem 2.2], so that the result of Proposition 3.8 is a special case of [7, Theorem 2.2]. 
We shall mainly work with properties of adjoint functors. Lemma 3.5 below seems to be a well-known result. It appears perhaps for the first time in the proof of [12, Lemma 0.4] without further reference. In order to keep the presentation as self-contained as possible, we shall provide a short proof.

Lemma 3.5. Let $\mathcal{C}, \mathcal{C}^{\prime}$ and $\mathcal{D}$ be categories, let $L: \mathcal{C} \longrightarrow \mathcal{C}^{\prime}$ be a functor admitting a right adjoint $R$, and let $\hat{L}$ be the induced functor $F$ unct $\left(\mathcal{C}^{\prime}, \mathcal{D}\right) \longrightarrow F$ unct $(\mathcal{C}, \mathcal{D})$ on the functor categories given by precomposition with $L$; likewise let $\hat{R}$ be the induced functor Funct $(\mathcal{C}, \mathcal{D}) \longrightarrow F$ unct $\left(\mathcal{C}^{\prime}, \mathcal{D}\right)$ given by precomposition with $R$. Then $\hat{R}$ is left adjoint to $\hat{L}$.

Proof. We get natural transformations

$$
i d_{\mathcal{C}} \stackrel{\eta}{\longrightarrow} R L \text { and } L R \stackrel{\epsilon}{\longrightarrow} i d_{\mathcal{C}^{\prime}}
$$

so that the compositions

$$
L \longrightarrow L R L \longrightarrow L \text { and } R \longrightarrow R L R \longrightarrow R
$$

are the identity on the respective ctegories (cf. e.g. Maclane [26, IV.1. Theorem 2]).

Now, given any functor $X: \mathcal{C} \longrightarrow \mathcal{D}$, we obtain a natural transformation

$$
i d_{\text {Funct }(\mathcal{C}, \mathcal{D})} \longrightarrow(\hat{L} \hat{R})
$$

induced from $\eta$ by

$$
X \stackrel{X(\eta)}{\longrightarrow} X R L=(\hat{L} \hat{R})(X)
$$

and likewise a natural transformation

$$
(\hat{R} \hat{L}) \longrightarrow i d_{\text {Funct }\left(\mathcal{C}^{\prime}, \mathcal{D}\right)} \text {. }
$$

Since the compositions $L \longrightarrow L R L \longrightarrow L$ and $R \longrightarrow R L R \longrightarrow R$ are the identity, this holds as well for the compositions $\hat{L} \longrightarrow \hat{L} \hat{R} \hat{L} \longrightarrow \hat{L}$ and $\hat{R} \longrightarrow$ $\hat{R} \hat{L} \hat{R} \longrightarrow \hat{R}$. Again by [26, IV.1. Theorem 2] we obtain the statement.

We shall need a very simple observation.

Lemma 3.6. Let $A$ and $B$ be two polynomial functors of degree at most $n$ and let

$$
0 \longrightarrow A \longrightarrow C \longrightarrow B \longrightarrow 0
$$

be an exact sequence of functors. Then, the degree of $C$ is at most $n$ as well. Moreover, taking cross effects is exact.

Proof. We get a commutative diagram

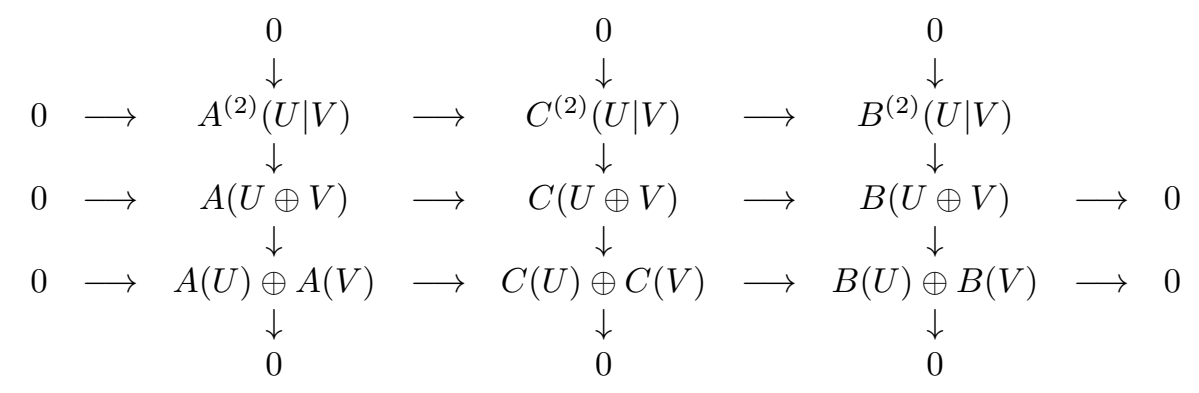


and the snake lemma implies that

$$
0 \longrightarrow A^{(2)}(U \mid V) \longrightarrow C^{(2)}(U \mid V) \longrightarrow B^{(2)}(U \mid V) \longrightarrow 0
$$

is exact. Induction on the degree gives the result.

We shall now prove a general statement on functor categories which is an adaption from Franjou [11, Section 1].

Lemma 3.7. Let $R$ be a commutative ring and let $G$ be an object in $\mathcal{A}_{R}^{n}$. Then we get $\operatorname{Hom}_{\mathcal{A}_{R}}\left(\left(R \otimes i d^{\otimes n}\right), G\right)=G^{(n-1)}$.

Proof. Let

$$
n-\mathbb{Z}-\text { free }=\underbrace{(\mathbb{Z}-\text { free }) \times \cdots \times(\mathbb{Z}-\text { free })}_{n \text { copies }}
$$

be the category with objects $F_{1} \times \cdots \times F_{n}$ for $F_{i}$ being an object in $\mathbb{Z}-$ free for each $i \in\{1, \ldots, n\}$ and morphisms being

$\operatorname{Hom}_{n-\mathbb{Z}-\text { free }}\left(F_{1} \times \cdots \times F_{n}, G_{1} \times \cdots \times G_{n}\right):=\operatorname{Hom}_{\mathbb{Z}}\left(F_{1}, G_{1}\right) \times \cdots \times \operatorname{Hom}_{\mathbb{Z}}\left(F_{n}, G_{n}\right)$

for all objects $F_{i}, G_{j}$ of $\mathbb{Z}-$ free. Composition of morphisms is given by composition of mappings in $\mathbb{Z}$ - free. We shall define

$$
\Pi_{n}: n-\mathbb{Z}-\text { free } \longrightarrow \mathbb{Z}-\text { free }
$$

and

$$
\Delta_{n}: \mathbb{Z}-\text { free } \longrightarrow n-\mathbb{Z}-\text { free }
$$

by $\Pi_{n}\left(F_{1}, \ldots, F_{n}\right):=F_{1} \oplus \cdots \oplus F_{n}$ and $\Delta_{n}(F):=(F, \ldots, F)$. Then $\Pi_{n}$ is left and right adjoint to $\Delta_{n}$ as is easily seen, almost by definition.

Now, we consider the category $n-\mathcal{A}_{R}$ of functors $n-\mathbb{Z}-$ free $\longrightarrow R-\operatorname{Mod}$ with morphisms being natural transformations. We get functors

$$
\begin{aligned}
n-\mathcal{A}_{R} & \stackrel{\Delta^{n}}{\longrightarrow} \mathcal{A}_{R} \\
F & \mapsto \quad F \circ \Delta_{n}
\end{aligned}
$$

and

$$
\begin{aligned}
\mathcal{A}_{R} & \stackrel{\Pi^{n}}{\longrightarrow} \quad n-\mathcal{A}_{R} \\
F & \mapsto \quad F \circ \Pi_{n} .
\end{aligned}
$$

By Lemma 3.5 we see that the functor $\Pi^{n}$ is left and right adjoint to $\Delta^{n}$. Put

$$
\begin{aligned}
& n-\mathbb{Z}-\text { free } \stackrel{\otimes^{n}}{\longrightarrow} \mathbb{Z} \text {-free } \\
& \left(M_{1}, \ldots, M_{n}\right) \quad \mapsto \quad M_{1} \otimes \cdots \otimes M_{n}
\end{aligned}
$$

and obtain $i d^{\otimes n}=\otimes^{n} \circ \Delta_{n}$ and $R \otimes i d^{\otimes n}=\left(R \otimes \otimes^{n}\right) \circ \Delta_{n}$. But then

$$
\begin{aligned}
\operatorname{Hom}_{\mathcal{A}_{R}}\left(\left(R \otimes i d^{\otimes n}\right), G\right) & =\operatorname{Hom}_{\mathcal{A}_{R}}\left(\left(R \otimes \nabla^{n}\right) \circ \Delta_{n}, G\right) \\
& =\operatorname{Hom}_{n-\mathcal{A}_{R}}\left(\left(R \otimes \nabla^{n}\right), G \circ \Pi_{n}\right) .
\end{aligned}
$$

Let $I:=\left\{i_{1}, \ldots, i_{m}\right\} \subseteq\{1, \ldots, n\}$ be an $m$-element subset of $\{1, \ldots, n\}$, and suppose $i_{1}<\cdots<i_{m}$. Then we may consider $\pi_{I}^{n}: n-\mathbb{Z}-$ free $\longrightarrow m-\mathbb{Z}-$ free to be the functor given by

$$
\pi_{I}^{n}\left(M_{1}, \ldots, M_{n}\right):=\left(M_{i_{1}}, \ldots, M_{i_{m}}\right)
$$


and the functor $\iota_{I}^{n}: m-\mathbb{Z}-$ free $\longrightarrow n-\mathbb{Z}-$ free given by injection into the corresponding coordinates so that $\pi_{I}^{n} \circ \iota_{I}^{n}=i d$. Then, again by definition, $\iota_{I}^{n}$ is left and right adjoint to $\pi_{I}^{n}$. Using Lemma 3.5 we get that the functors

$$
\widehat{\iota_{I}^{n}}: n-\mathcal{A}_{R} \longrightarrow m-\mathcal{A}_{R}
$$

and

$$
\widehat{\pi_{I}^{n}}: m-\mathcal{A}_{R} \longrightarrow n-\mathcal{A}_{R}
$$

obtained by pre-composition with the functors $\iota_{I}^{n}$ and $\pi_{I}^{n}$ form a pair of left and right adjoint functors.

By definition of the cross effect of a functor we get

$$
G\left(V_{1} \oplus \cdots \oplus V_{n}\right)=\bigoplus_{m=1}^{n} \bigoplus_{i_{1}<\cdots<i_{m}} G^{(m-1)}\left(V_{i_{1}}|\ldots| V_{i_{m}}\right) .
$$

Hence

$$
G \circ \Pi_{n}=\bigoplus_{m=1}^{n} \bigoplus_{i_{1}<\cdots<i_{m}} G^{(m-1)} \circ \pi_{\left\{i_{1}, \ldots, i_{m}\right\}}^{n} .
$$

Moreover, using that $\widehat{\iota_{I}^{n}}$ is left adjoint to $\widehat{\pi_{I}^{n}}$, in case $m \neq n$ we get

$$
\begin{aligned}
& \operatorname{Hom}_{n-\mathcal{A}_{R}}\left(\left(R \otimes \nabla^{n}\right), G^{(m-1)} \circ \pi_{\left\{i_{1}, \ldots, i_{m}\right\}}^{n}\right) \\
& \quad=\operatorname{Hom}_{n-\mathcal{A}_{R}}\left(\left(R \otimes \nabla^{n}\right) \circ \iota_{\left\{i_{1}, \ldots, i_{m}\right\}}^{n}, G^{(m-1)}\right)=0
\end{aligned}
$$

since at least one of the factors in the tensor product is 0 . Therefore

$$
H o m_{\mathcal{A}_{R}}\left(\left(R \otimes i d^{\otimes n}\right), G\right)=H_{o m-\mathcal{A}_{R}}\left(\left(R \otimes \otimes^{n}\right), G^{(n-1)}\right) .
$$

Since $G$ is of degree at most $n$, the functor $G^{(n-1)}$ is additive in each variable. Hence its value is given by the $R$-module $G^{(n-1)}(\mathbb{Z}|\ldots| \mathbb{Z})$. However, we readily verify that $\left(R \otimes i d^{\otimes^{n}}\right)(\mathbb{Z}|\ldots| \mathbb{Z})=R$ and $\operatorname{Hom}_{R}\left(R, G^{(n-1)}(\mathbb{Z}|\ldots| \mathbb{Z})\right)=G^{(n-1)}(\mathbb{Z}|\ldots| \mathbb{Z})$. Hence a natural transformation in $\operatorname{Hom}_{n-\mathcal{A}_{R}}\left(\left(R \otimes \mathbb{\nabla}^{n}\right), G^{(n-1)}\right)$ induces an element in $G^{(n-1)}(\mathbb{Z}|\ldots| \mathbb{Z})$. On the other hand, any element in $G^{(n-1)}(\mathbb{Z}|\ldots| \mathbb{Z})$ induces a natural transformation in $\operatorname{Hom}_{n-\mathcal{A}_{R}}\left(\left(R \otimes \bigotimes^{n}\right), G^{(n-1)}\right)$. Therefore

$$
\operatorname{Hom}_{\mathcal{A}_{R}}\left(R \otimes i d^{\otimes n}, G\right)=G^{(n-1)},
$$

as claimed, and we obtain the statement.

\section{Proposition 3.8.}

$$
\begin{aligned}
& \leftarrow \mathcal{A}_{\mathbb{F}_{p}}^{n-1} \rightarrow \mathcal{A}_{\mathbb{F}_{p}}^{n} \stackrel{\leftarrow}{\stackrel{e}{\rightarrow}} \mathbb{F}_{p} \mathfrak{S}_{n}-\bmod \\
\leftarrow & \leftarrow
\end{aligned}
$$

is a recollement diagram with

$$
e:=\operatorname{Hom}_{\mathcal{A}_{\mathbb{F}_{p}}}(\mathbb{F}_{p} \otimes_{\mathbb{Z}} \underbrace{i d \otimes_{\mathbb{Z}} \cdots \otimes_{\mathbb{Z}} i d}_{n \text { factors }},-): \mathcal{A}_{\mathbb{F}_{p}}^{n} \longrightarrow \mathbb{F}_{p} \mathfrak{S}_{n}-\text { mod. }
$$

Proof. We shall show the following auxiliary lemma needed for the proof of the proposition.

Lemma 3.9. $\mathbb{F}_{p} \otimes i d^{\otimes n}$ is projective and injective in $\mathcal{A}_{\mathbb{F}_{p}}^{n}$. 
Proof. Taking cross effects is exact. By Lemma 3.7 we see that $\mathbb{F}_{p} \otimes i d^{\otimes n}$ is projective. Using that the duality $D$ on functors $F: \mathbb{Z}-$ free $\longrightarrow \mathbb{F}_{p}-\operatorname{Mod}$ is given by

$$
(D F)(V)=H_{o m} \mathbb{F}_{p}\left(F\left(H o m_{\mathbb{Z}}(V, \mathbb{Z})\right), \mathbb{F}_{p}\right),
$$

and observing that $\mathbb{F}_{p} \otimes i d^{\otimes n}$ is self-dual, we obtain that $\mathbb{F}_{p} \otimes i d^{\otimes n}$ is injective as well.

We need to prove that

$$
\mathcal{A}_{\mathbb{F}_{p}}^{n-1}=\left\{F \in \mathcal{A}_{\mathbb{F}_{p}}^{n} \mid H o m_{\mathcal{A}_{\mathbb{F}_{p}}}\left(\mathbb{F}_{p} \otimes i d^{\otimes n}, F\right)=0\right\} .
$$

But this is clear by Lemma 3.7

Since $\mathbb{F}_{p} \otimes i d^{\otimes n}$ is projective there is a right adjoint and a left adjoint to $H \operatorname{Hom}_{\mathcal{A}_{\mathbb{F}_{p}}}\left(\mathbb{F}_{p} \otimes i d^{\otimes n},-\right)$, namely the functor $M \mapsto\left(\mathbb{F}_{p} \otimes i d^{\otimes n} \otimes M\right)^{\mathfrak{S}_{n}}$ is the right adjoint and the functor $M \mapsto\left(\mathbb{F}_{p} \otimes i d^{\otimes n} \otimes M\right)_{\mathfrak{S}_{n}}$ is the left adjoint.

Moreover, the unit and the counit of the adjunctions induce the identity on $\mathbb{F}_{p} \mathfrak{S}_{n}$-mod. This can be done literally as in Piriou [30, Proposition 2.2.2].

This shows Proposition 3.8

As a consequence we show the following lemma.

Lemma 3.10. Any simple object in $\mathcal{A}_{\mathbb{F}_{p}}^{n}$ is in the image of $\circ\left(\mathbb{F}_{p} \otimes-\right): \mathcal{F}_{\mathbb{F}_{p}}^{n} \hookrightarrow \mathcal{A}_{\mathbb{F}_{p}}^{n}$, and any simple object of $\mathcal{A}_{\mathbb{F}_{p}}^{n}$ gives a simple object in $\mathcal{F}_{\mathbb{F}_{p}}^{n}$ this way.

Proof. We shall use induction on $n$. There is a morphism of recollement diagrams as follows, where the vertical functors are fully faithful embeddings of categories by Lemma 1.2

$$
\begin{array}{rlccc} 
& \leftarrow & & \stackrel{\leftarrow}{\leftarrow} & \\
\mathcal{F}_{\mathbb{F}_{p}}^{n-1} & \rightarrow & \mathcal{F}_{\mathbb{F}_{p}}^{n} & \stackrel{e_{\mathcal{F}}^{n}}{\rightarrow} & \mathbb{F}_{p} \mathfrak{S}_{n}-\bmod \\
& \leftarrow & & \leftarrow & \\
\downarrow i_{n-1} & & \downarrow i_{n} & & \| \\
& & & & \\
\mathcal{A}_{\mathbb{F}_{p}}^{n-1} & \rightarrow & & \stackrel{\leftarrow}{\leftarrow} & \\
& \leftarrow & & \stackrel{e_{\mathbb{A}_{p}^{n}}}{\longrightarrow} & \mathbb{F}_{p} \mathfrak{S}_{n}-\bmod \\
& \leftarrow &
\end{array}
$$

Therefore the number of simple objects in $\mathcal{A}_{\mathbb{F}_{p}}^{n}$ and in $\mathcal{F}_{\mathbb{F}_{p}}^{n}$ coincides.

The statement is clear for $n \leq p-1$ by Lemma 1.6. Let $n \geq p$ and let $S$ be a simple object in $\mathcal{F}_{\mathbb{F}_{p}}^{n}$. We may suppose, using the induction hypothesis, that $e_{\mathcal{F}^{n}}(S) \neq 0$. Then, suppose $X$ is a simple and proper subobject of $i_{n} S$. Since $e_{\mathcal{A}^{n}}$ is exact, $e_{\mathcal{A}^{n}}(X)$ is a subobject of $e_{\mathcal{A}^{n}}\left(i_{n} S\right)=e_{\mathcal{F}^{n}}(S)$. Since $e_{\mathcal{F}^{n}}$ is exact, $e_{\mathcal{F}^{n}}(S)$ is simple. So, $e_{\mathcal{A}^{n}}(X)$ is either 0 or isomorphic to $e_{\mathcal{F}^{n}}(S)$. Since $X$ is a proper subobject of $i_{n} S$ we see that $e_{\mathcal{A}^{n}}(X)=0$, and hence $X \in \mathcal{A}^{n-1}$. Since the simple objects of $\mathcal{F}^{n-1}$ and of $\mathcal{A}^{n-1}$ coincide by the induction hypothesis, $X$ is a proper non-zero subobject of $S$. This gives the contradiction.

We have seen in Lemma 1.6 and Remark 3.2 that

$$
\mathcal{F}_{\mathbb{F}_{p}}^{p-1} \simeq \mathcal{A}_{\mathbb{F}_{p}}^{p-1} \simeq \prod_{n<p} \prod_{\lambda \vdash n} \mathbb{F}_{p}-\bmod
$$




\section{Short REVIEW of Brauer TREe ALGEBras}

Proposition 3.8 shows that the representation theory of the symmetric group is closely related to $\mathcal{A}^{n}(p)$; we shall need some information from group representations. We give Benson [4, Section 4.18 and Section 6.5] as a general reference.

Let $k$ be a field of characteristic $p>0$ and let $G$ be a finite group so that $k$ is a splitting field for $G$, i.e. the endomorphism ring of each simple $k G$-module is $k$. Then it is well-known that there are only a finite number of isomorphism classes of indecomposable $k G$-modules if and only if the Sylow $p$ subgroups of $G$ are all cyclic. In particular, the symmetric group $\mathfrak{S}_{p}$ is a group with every Sylow $p$ subgroup being cyclic.

An indecomposable ring-direct factor of $k G$ is called a $p$-block of $G$, and the representation theory of a block $B$ is completely understood in case the Sylow $p$ subgroups of $G$ are cyclic. The algebras occurring in this case are so-called Brauer tree algebras.

We explain briefly the theory of Brauer tree algebras and refer to Benson [4, Section 4.18 and Section 6.5] or to Auslander-Reiten-Smalø [1, Section X.3] for more details.

A Brauer tree is a finite connected tree $\Gamma$, i.e. a connected graph without cycles and multiple edges, together with an additional structure as we shall describe now: Let $\Gamma_{0}$ be the vertices of $\Gamma$ and let $\Gamma_{1}$ be the edges of $\Gamma$. For each $e \in \Gamma_{1}$ let $v_{1}(e) \in \Gamma_{0}$ and $v_{2}(e) \in \Gamma_{0}$ be the vertices adjacent to $e$.

- Then for each vertex $v \in \Gamma_{0}$ let $E_{v}$ be the edges adjacent to $v$, and suppose given a transitive permutation $\sigma_{v} \in \mathfrak{S}_{E_{v}}$.

- Moreover, we choose one vertex $v_{0} \in \Gamma_{v}$ and an integer $\mu>0$, called the exceptional multiplicity.

A Brauer tree algebra $B$ is then a finite-dimensional symmetric $k$-algebra so that

- the isomorphism classes of simple $B$-modules are parameterised by $\Gamma_{1}$, denoting by $S_{e}$ a simple $B$-module whose isomorphism class corresponds to the edge $e \in \Gamma_{1}$,

- the projective cover $P_{e}$ of $S_{e}$ has the property that $\operatorname{rad}\left(P_{e}\right) / \operatorname{soc}\left(P_{e}\right)=$ $U_{v_{1}(e)} \oplus U_{v_{2}(e)}$, where $U_{v_{1}(e)}$ and $U_{v_{2}(e)}$ are both uniserial modules,

- for each $j \in\{1,2\}$,

- if $v_{j}(e)$ is not the exceptional vertex, then the composition length of $U_{v_{j}(e)}$ is equal to $\left|E_{v_{j}(e)}\right|-1$,

- if $v_{j}(e)$ is the exceptional vertex, then the composition length of $U_{v_{j}(e)}$ is equal to $\mu \cdot\left|E_{v_{j}(e)}\right|-1$,

- for each $i$, for which the $i$-th radical quotient is not 0 ,

$$
\operatorname{rad}^{i}\left(U_{v_{1}(e)}\right) / \operatorname{rad}^{i+1}\left(U_{v_{1}(e)}\right) \simeq S_{\sigma_{v_{1}(e)}^{i+1}(e)}
$$

and

$$
\operatorname{rad}^{i}\left(U_{v_{2}(e)}\right) / \operatorname{rad}^{i+1}\left(U_{v_{2}(e)}\right) \simeq S_{\sigma_{v_{2}(e)}^{i+1}(e)} .
$$

One observes that in case $\mu=1$, there is no difference between the exceptional vertex and a non-exceptional vertex. Hence, in this case we do not need to fix an exceptional vertex, and we say that the Brauer tree has no exceptional vertex if the exceptional multiplicity $\mu$ is 1 .

Observe further that if a vertex $v$ is a leaf, i.e. $E_{v}=\{e\}$, then the projective cover of $S_{e}$ is uniserial and $U_{v}=0$. 
A particular case is when each vertex has at most 2 edges adjacent to it, or in other words if $\left|E_{v}\right| \leq 2$ for all $v \in \Gamma_{0}$. In this case we call the Brauer tree a stem. If moreover the Brauer tree has no exceptional vertex, then the tree can be visualised as

The projective indecomposable modules $P$ are then all of Loewy length 3 , i.e. $P$ has a simple top, a simple socle and $\operatorname{rad}(P) / \operatorname{soc}(P)$ is semisimple of composition length at most 2 .

This situation occurs for $\mathbb{F}_{p} \mathfrak{S}_{p}$ which has several blocks for $p \geq 5$, one of which is a Brauer tree algebra associated to a stem without exceptional vertex and $p-1$ edges, and all the other blocks are simple algebras.

\section{The Structure of POlynomial Functors MOdulo $p$}

The situation of polynomial functors of degree $p$ is different from those of degree $n<p$. In this section we are going to describe their structure completely. From now on we assume that $p \geq 5$ since the representation theory of $\mathbb{F}_{2} \mathfrak{S}_{2}$ and of $\mathbb{F}_{3} \mathfrak{S}_{3}$ is slightly different from the case $p \geq 5$.

Remark 5.1. Let us recall the relation between the Schur algebra $S_{\mathbb{F}_{p}}(p, p)$ and the group algebra $\mathbb{F}_{p} \mathfrak{S}_{p}$. We shall use the structure of the Schur algebra and the corresponding Hecke algebra as it is proved in [20, Corollary 1.3]. The group algebra of the symmetric group is a special case of the Hecke algebra. For general information on Schur algebras and Brauer tree algebras see [17] and [21. The algebra $S_{\mathbb{F}_{p}}(p, p)$ admits $p$ simple modules $S_{1}, \ldots, S_{p-1}, S_{p}$, whereas the group algebra $\mathbb{F}_{p} \mathfrak{S}_{p}$ admits $p-1$ simple modules $S_{1}^{\prime}, \ldots, S_{p-1}^{\prime}$. The projective indecomposable $S_{\mathbb{F}_{p}}(p, p)$-modules have composition series

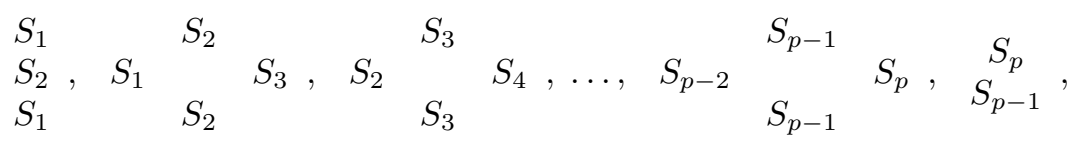

whereas the projective indecomposable $\mathbb{F}_{p} \mathfrak{S}_{p}$-modules have composition series

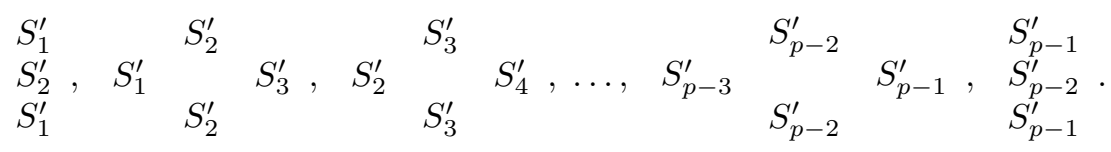

Lemma 5.2. Let $L$ be the simple polynomial functor in $\mathcal{F}_{\mathbb{F}_{p}}^{p}$ so that $L$ is of degree $p$ and so that $L$ corresponds to the trivial representation of $\mathbb{F}_{p} \mathfrak{S}_{p}$. Then, $\operatorname{Ext}_{\mathcal{F}_{\mathbb{F}_{p}}}^{1}(i d, L) \neq 0 \neq \operatorname{Ext}_{\mathcal{F}_{\mathbb{F}_{p}}}^{1}(L, i d)$. Moreover, if $\operatorname{Ext}_{\mathcal{F}_{\mathbb{F}_{p}}}^{1}(i d, S) \neq 0$ or $0 \neq$ $\operatorname{Ext}_{\mathcal{F}_{\mathbb{F}_{p}}}^{1}(S, i d)$ for a simple degree $p$-functor $S$, then $L \simeq S$.

Proof. Let $S_{1}$ be the simple polynomial functor of degree 1. The identity functor $i d$ is trivially of degree 1 and simple, which implies $S_{1}=i d$. Then, $\operatorname{Ext}_{\mathcal{F}^{p}}^{1}(L, i d)$ is not necessarily zero for $L$ being an irreducible polynomial functor of degree $p$. Now, since we are working over $\mathbb{F}_{p}$, we get that $i d^{(1)} \simeq i d$ as a polynomial functor, but not as a strict polynomial functor. As a strict polynomial functor, $I^{(1)}$ is of degree $p$.

Theorem 2.5 implies that there is only one simple functor $L$ of degree $p$ with $\operatorname{Ext}_{\mathcal{F}^{p}}^{1}(L, i d) \neq 0$. Proposition 2.3 in connection with Theorem 2.2 imply that $L$ is the simple functor corresponding to the trivial $\mathbb{F}_{p} \mathfrak{S}_{p}$-module, since this is the 
module which has an extension with the unique simple module of the Schur algebra $S_{\mathbb{F}_{p}}(p, p)$ which is not a simple $\mathbb{F}_{p} \mathfrak{S}_{p}$-module (cf. Remark 5.1).

This implies that

$$
\operatorname{Ext}_{\mathcal{P}(p)}^{1}\left(L, i d^{(1)}\right) \simeq \operatorname{Ext}_{\mathcal{F}_{\mathbb{F}_{p}}}^{1}\left(L, i d^{(1)}\right) \simeq \operatorname{Ext}_{\mathcal{F}_{\mathbb{F}_{p}}}^{1}(L, i d)
$$

Since the category of degree $p$ strict polynomial functors is equivalent to the category of modules over the Schur algebra $S_{\mathbb{F}_{p}}(p, p)$, one sees that

$$
\operatorname{Ext}_{\mathcal{P}_{\mathbb{F}_{p}}}^{1}\left(L, i d^{(1)}\right) \simeq \operatorname{Ext}_{S_{\mathbb{F}_{p}}(p, p)}^{1}\left(V, I_{0}\right)
$$

for $I_{0}$ being the simple $S_{\mathbb{F}_{p}}(p, p)$-module corresponding to the $p$ singular partition of $p$ and $V$ being the simple $S_{\mathbb{F}_{p}}(p, p)$-module corresponding to $L$. Finally, it is a classical fact (cf. e.g. [20]) that $\operatorname{Ext}_{S_{\mathbb{F}_{p}}(p, p)}^{1}\left(I_{0}, V\right) \neq 0$ or $\operatorname{Ext}_{S_{\mathbb{F}_{p}}(p, p)}^{1}\left(V, I_{0}\right) \neq 0$ if and only if $V$ corresponds to the trivial module of the symmetric group, and in this case, the dimension of $\operatorname{Ext}_{S_{\mathbb{F}_{p}}(p, p)}^{1}\left(I_{0}, V\right)$ and of $\operatorname{Ext}_{S_{\mathbb{F}_{p}}(p, p)}^{1}\left(V, I_{0}\right)$ is 1 . This proves the statement.

Using the embedding $\mathcal{F}_{\mathbb{F}_{p}}^{p} \hookrightarrow \mathcal{A}_{\mathbb{F}_{p}}^{p}$ from Lemma 1.2, the functor $L$ of $\mathcal{F}_{\mathbb{F}_{p}}^{p}$ induces a functor $L\left(\mathbb{F}_{p} \otimes-\right)$ in $\mathcal{A}_{\mathbb{F}_{p}}^{p}$. In order to avoid additional notational burden we shall denote this functor $L\left(\mathbb{F}_{p} \otimes-\right)$ by $L$ as well.

We get as a corollary the following statement.

Corollary 5.3. $\operatorname{Ext}_{\mathcal{A}_{\mathbb{F}_{p}}^{p}}^{1}\left(\mathbb{F}_{p} \otimes_{\mathbb{Z}} i d, L\right) \neq 0 \neq \operatorname{Ext}_{\mathcal{A}_{\mathbb{F}_{p}}^{p}}^{1}\left(L, \mathbb{F}_{p} \otimes_{\mathbb{Z}} i d\right)$.

Proof. We know that $E x t_{\mathcal{F}_{\mathbb{F}_{p}}^{p}}^{1}(i d, L) \neq 0$. So, there is a non-split exact sequence

$$
0 \longrightarrow L \longrightarrow X \longrightarrow i d \longrightarrow 0
$$

for some functor $X$ in $\mathcal{F}_{\mathbb{F}_{p}}^{p}$, since $\mathcal{F}_{\mathbb{F}_{p}}^{p} \hookrightarrow \mathcal{A}_{\mathbb{F}_{p}}^{p}$ by Lemma 1.2, This induces an exact sequence

$$
0 \longrightarrow L\left(\mathbb{F}_{p} \otimes_{\mathbb{Z}} i d\right) \longrightarrow X\left(\mathbb{F}_{p} \otimes_{\mathbb{Z}} i d\right) \longrightarrow \mathbb{F}_{p} \otimes_{\mathbb{Z}} i d \longrightarrow 0
$$

in $\mathcal{A}_{\mathbb{F}_{p}}^{p}$, where $L\left(\mathbb{F}_{p} \otimes_{\mathbb{Z}} i d\right)$ is simple by Lemma 3.10. This sequence is non-split since the functor pre-composing with $\mathbb{F}_{p} \otimes_{\mathbb{Z}} i d$ is a fully faithful embedding. Hence $E x t_{\mathcal{A}_{\mathbb{F}_{p}}^{p}}^{1}\left(\mathbb{F}_{p} \otimes_{\mathbb{Z}} i d, L\right) \neq 0$. Therefore, $L$ is a direct factor of the top of the radical of the projective cover of $\mathbb{F}_{p} \otimes_{\mathbb{Z}} i d$. Similarly, $\operatorname{Ext}_{\mathcal{A}_{\mathbb{F}_{p}}^{p}}^{1}\left(L, \mathbb{F}_{p} \otimes_{\mathbb{Z}} i d\right) \neq 0$.

Actually, the argument of Lemma 5.2 gives another slightly different statement.

Lemma 5.4. Let $X$ and $Y$ be two simple functors of degree at most $p$. Then,

$$
\operatorname{Ext}_{\mathcal{F}_{\mathbb{F}_{p}}^{p}}^{1}(X, Y) \neq 0 \Rightarrow \operatorname{deg}(X)-\operatorname{deg}(Y) \in\{0, p-1\} .
$$

Proof. By Lemma 3.10 we know that the simple functors $X$ and $Y$ can be considered to lie in $\mathcal{F}_{\mathbb{F}_{p}}^{p}$. Proposition 2.3 in connection with Theorem 2.2 imply this result.

Remark 5.5. At the present stage it might happen that $\operatorname{Ext}_{\mathcal{A}_{\mathbb{F}_{p}}^{p}}^{1}(X, Y) \neq 0$ even though $\operatorname{Ext}_{\mathcal{F}_{\mathbb{F}_{p}}^{p}}^{1}(X, Y)=0$.

Denoting by $\rho_{p}(i)$ the number of $p$-regular partitions of $i$, the algebra $\Gamma_{\mathbb{F}_{p}}^{p}$ is Morita equivalent to a direct product of $\left(\sum_{i=1}^{p-1} \rho_{p}(i)\right)-1$ copies of $\mathbb{F}_{p}$ and of an indecomposable ring $\Gamma_{\mathbb{F}_{p}, 0}^{p}$. By the recollement diagram preceding Lemma 3.3 this 
ring $\Gamma_{\mathbb{F}_{p}, 0}^{p}$ has a projective module $P=\Gamma_{\mathbb{F}_{p}, 0}^{p} \cdot e$ so that the endomorphism ring of $P$ is Morita equivalent to the Brauer tree algebra corresponding to $\mathbb{F}_{p} \mathfrak{S}_{p}$. There is a projective indecomposable $\Gamma_{\mathbb{F}_{p}, 0}^{p}$-module $P_{0}$ so that $P_{0} \oplus P$ is a progenerator of $\Gamma_{\mathbb{F}_{p}, 0}^{p}$, and the endomorphism ring of $P_{0} \oplus P$ is basic and Morita equivalent to $\Gamma_{\mathbb{F}_{p}, 0}^{p}$. Moreover, $\Gamma_{\mathbb{F}_{p}, 0}^{p} /\left(\Gamma_{\mathbb{F}_{p}, 0}^{p} \cdot e \cdot \Gamma_{\mathbb{F}_{p}, 0}^{p}\right)$ is Morita equivalent to $\mathbb{F}_{p}$.

The next remark constructs appropriate projective objects.

Let $R$ and $S$ be commutative rings. The functor $R[-] /\left(I^{n+1}\right)$ assigns to an $S$ module $V$ the quotient of the semigroup ring $R[V]$ on $V$ by the $n+1$-st power of the augmentation ideal. This functor $S-$ free $\longrightarrow R-\bmod$ is polynomial of degree $n$. Define $\operatorname{proj}_{n}^{m}:=\mathbb{F}_{p}\left[\operatorname{Hom}_{\mathbb{Z}}\left(\mathbb{Z}^{m},-\right)\right] /\left(I^{n+1}\right)$ and $\operatorname{proj}_{\infty}^{m}:=\mathbb{F}_{p}\left[\operatorname{Hom}_{\mathbb{Z}}\left(\mathbb{Z}^{m},-\right)\right]$.

Lemma 5.6. The functor proj ${ }_{n}^{m}$ in $\mathcal{A}_{\mathbb{F}_{p}}^{n}$ is projective and contains a projective cover of the reduction modulo $p$ functor $\mathbb{F}_{p} \otimes_{\mathbb{Z}} i d$.

Proof. For any degree $n$ polynomial functor $F$ one gets $\operatorname{Hom}_{\mathcal{A}_{\mathbb{F}_{p}}}\left(\operatorname{proj}_{n}^{m}, F\right) \simeq$ $F\left(\mathbb{Z}^{m}\right)$. This is true if one does not factorize the power of the augmentation ideal, and since all functors are of degree at most $n$, each natural transformation from $\operatorname{proj}_{\infty}^{m}$ to $F$ is zero on $I^{n+1}$ (see [12, Section 1]).

So, $\operatorname{Hom}_{\mathcal{A}}\left(\operatorname{proj}_{n}^{m},-\right)$ is exact, as the evaluation on exact sequences of functors is exact. Hence, $\operatorname{proj}_{n}^{m}$ is a projective object in $\mathcal{A}_{\mathbb{F}_{p}}^{n}$, and since $\operatorname{Hom}_{\mathcal{A}}\left(\operatorname{proj}_{n}^{m}, i d\right)=$ $i d\left(\mathbb{Z}^{m}\right)=\mathbb{Z}^{m} \neq 0$, the projective cover of the reduction modulo $p$ functor is a direct summand in $\operatorname{proj}_{n}^{m}$.

Remark 5.7. The situation is different for $\mathcal{F}_{\mathbb{F}_{p}}$. Indeed, we get that the functor $\mathbb{F}_{p}\left[\operatorname{Hom}_{\mathbb{F}_{p}}\left(\mathbb{F}_{p}^{m},-\right)\right] / I^{n+1}$ is projective in $\mathcal{F}_{\mathbb{F}_{p}}^{n}$ again since

$$
\operatorname{Hom}_{\mathcal{F}_{\mathbb{F}_{p}}^{n}}\left(\mathbb{F}_{p}\left[\operatorname{Hom}_{\mathbb{F}_{p}}\left(\mathbb{F}_{p}^{m},-\right)\right] / I^{n+1}, F\right) \simeq F\left(\mathbb{F}_{p}^{m}\right) .
$$

But, automatically $I^{p}=0$ for $m=1$ and the evaluation at $\mathbb{F}_{p}$ in this case, so that the endomorphism ring of $\mathbb{F}_{p}\left[\operatorname{Hom}_{\mathbb{F}_{p}}\left(\mathbb{F}_{p},-\right)\right] / I^{n+1}$ is an $\ell$-dimensional vector space where $\ell=\min (p, n)$.

Recall the embedding $\mathcal{F}_{\mathbb{F}_{p}}^{n} \longrightarrow \mathcal{A}_{\mathbb{F}_{p}}^{n}$ given by pre-composing with $\mathbb{F} \otimes_{\mathbb{Z}} i d$. The image of $\mathbb{F}_{p}\left[\operatorname{Hom}_{\mathbb{F}_{p}}\left(\mathbb{F}_{p},-\right)\right] / I^{n+1}$ under this embedding is

$$
\mathbb{F}_{p}\left[\operatorname{Hom}_{\mathbb{F}_{p}}\left(\mathbb{F}_{p}, \mathbb{F}_{p} \otimes_{\mathbb{Z}}-\right)\right] / I^{n+1},
$$

which is different from $\mathbb{F}_{p}\left[\operatorname{Hom}_{\mathbb{Z}}(\mathbb{Z},-)\right] / I^{n+1}$. As we will see, the projective indecomposable cover of $\mathbb{F}_{p} \otimes_{\mathbb{Z}}$ id in $\mathcal{A}_{\mathbb{F}_{p}}^{p}$ is a direct factor of the functor $\mathbb{F}_{p}\left[\operatorname{Hom}_{\mathbb{Z}}(\mathbb{Z},-)\right] / I^{p+1}$, and this is the only indecomposable projective functor which is not in the image of the embedding $\mathcal{F}_{\mathbb{F}_{p}}^{p} \longrightarrow \mathcal{A}_{\mathbb{F}_{p}}^{p}$.

Lemma 5.8. The projective functor proj $_{n}^{1}$ has one composition factor of degree $d$ for each $0 \leq d \leq n$ for all $n \leq p-1$. In particular, proj $_{n}^{1}$ contains a simple constant functor as a direct summand.

Proof. First, $\operatorname{proj}_{n}^{1}(0)=\mathbb{F}_{p}$, and so the simple functor of degree 0 is a direct factor of $\operatorname{proj}_{n}^{1}$.

Furthermore, $\operatorname{proj}_{n}^{1} \longrightarrow \operatorname{proj}_{n-1}^{1}$ for trivial reasons. Moreover, $\operatorname{proj}_{n}^{1}$ is of degree $n$ and not of degree $n-1$. We compute that $\operatorname{End}_{\mathcal{A}_{\mathbb{P}_{p}}^{n}}\left(\operatorname{proj}_{n}^{1}\right)=\operatorname{proj}_{n}^{1}(\mathbb{Z})=$ $\mathbb{F}_{p}[\mathbb{Z}] / I^{n+1}$ is an $n+1$-dimensional vector space. Moreover, by Lemma 1.6 and 
Remark 3.2 the projective module $\operatorname{proj}_{p-1}^{1}$ is semisimple since $\mathcal{A}_{\mathbb{F}_{p}}^{p-1}$ is a semisimple category. Observe that $\operatorname{proj}_{n}^{1}$ has exactly one composition factor more than $\operatorname{proj}_{n-1}^{1}$. This composition factor is of degree $n$ since

$$
\operatorname{Hom}_{\mathcal{A}_{\mathbb{P}_{p}}^{n}}(\operatorname{proj}_{n}^{1}, \mathbb{F}_{p} \otimes_{\mathbb{Z}} \underbrace{i d \otimes_{\mathbb{Z}} \cdots \otimes_{\mathbb{Z}} i d}_{n \text { factors }})=\mathbb{F}_{p} \otimes_{\mathbb{Z}} \mathbb{Z} \otimes_{\mathbb{Z}} \cdots \otimes_{\mathbb{Z}} \mathbb{Z}=\mathbb{F}_{p}
$$

and since $\mathbb{F}_{p} \otimes_{\mathbb{Z}} \underbrace{i d \otimes_{\mathbb{Z}} \cdots \otimes_{\mathbb{Z}} i d}_{n \text { factors }}$ is the projective object corresponding to degree $n$ polynomial functors in the recollement diagram.

Recall that $L$ denotes the simple functor in $\mathcal{A}_{\mathbb{F}_{p}}^{p}$ mapping to the trivial $\mathbb{F}_{p} \mathfrak{S}_{p^{-}}$ module in the recollement diagram.

Proposition 5.9. Suppose $p \geq 5$. The projective cover $P_{\mathbb{F}_{p} \otimes i d}$ of $\mathbb{F}_{p} \otimes_{\mathbb{Z}}$ id in $\mathcal{A}_{\mathbb{F}_{p}}^{p}$ is uniserial with top and socle being $\mathbb{F}_{p} \otimes_{\mathbb{Z}}$ id and with $\operatorname{rad}\left(P_{\mathbb{F}_{p} \otimes_{\mathbb{Z}} i d}\right) / \operatorname{soc}\left(P_{\mathbb{F}_{p} \otimes_{\mathbb{Z}} i d}\right) \simeq L$. Moreover,

$$
\operatorname{proj}_{p}^{1} \simeq S_{0} \oplus P_{\mathbb{F}_{p} \otimes i d} \oplus S_{2} \oplus \cdots \oplus S_{p-1}
$$

for simple functors $S_{i}$ of degree $i$.

Proof. We shall divide the proof into various claims.

Claim 5.10. No direct summand of the top of $\operatorname{proj}_{p}^{1}$ is of degree $p$.

Proof. We have $\operatorname{Hom}_{\mathcal{A}_{\mathbb{F}_{p}}^{p}}\left(\operatorname{proj}_{p}^{1},\left(\mathbb{F}_{p} \otimes_{\mathbb{Z}} i d\right)^{\otimes p}\right)=\mathbb{F}_{p}$. On the other hand, we know from the recollement diagram that $\left(\mathbb{F}_{p} \otimes_{\mathbb{Z}} i d\right)^{\otimes p}$ is the projective cover of the simple modules coming from $\mathbb{F}_{p} \mathfrak{S}_{p}$, that is, those of degree $p$. Now, each projective indecomposable module of $\mathbb{F}_{p} \mathfrak{S}_{p}$ has the property that the top of this module is isomorphic to the socle of this module and that the top and the socle of this projective indecomposable module are different. Hence, suppose a simple polynomial functor of degree $p$ would be in the top of $\operatorname{proj}_{p}^{1}$; then let $Q$ be its projective cover in $\mathcal{A}_{\mathbb{F}_{p}}^{p}$. Further, $Q$ is a direct summand of $\left(\mathbb{F}_{p} \otimes_{\mathbb{Z}} i d\right)^{\otimes p}$. Since the top of $\left(\mathbb{F}_{p} \otimes_{\mathbb{Z}} i d\right)^{\otimes p}$ is isomorphic to the socle of $\left(\mathbb{F}_{p} \otimes_{\mathbb{Z}} i d\right)^{\otimes p}$, the above homomorphism space would be at least 2-dimensional, corresponding to the mapping of $\operatorname{proj}_{p}^{1}$ on the top and on the socle of $\left(\mathbb{F}_{p} \otimes_{\mathbb{Z}} i d\right)^{\otimes p}$.

Lemma 5.11. If $\operatorname{Ext}_{\mathcal{A}_{\mathbb{F}_{p}}^{p}}^{1}(S, T) \neq 0$ for two simple functors $S$ and $T$, then $\operatorname{deg}(S)-$ $\operatorname{deg}(T) \in\{0, p-1\}$, and if $\operatorname{deg}(S)=\operatorname{deg}(T)$, then $\operatorname{deg}(S)=p$.

Proof. We know by Lemma 1.6 that $\mathcal{A}_{\mathbb{F}_{p}}^{p-1}$ is semisimple. Moreover, the category of constant functors is a direct factor in the category of polynomial functors. Using Lemma 3.6 this shows the statement.

We denote by $P_{V}$ the projective cover of the functor $V$ in $\mathcal{A}_{\mathbb{F}_{p}}^{p}$.

Claim 5.12. proj $_{p}^{1} \simeq S_{0} \oplus S_{2} \oplus S_{3} \oplus \cdots \oplus S_{p-1} \oplus M$ for simple projective functors $S_{i}$ of degree $i$ and the projective cover $M$ of the functor $\mathbb{F}_{p} \otimes_{\mathbb{Z}} i d$.

Proof. $\operatorname{End}_{\mathcal{A}_{\mathbb{F}_{p}}^{p}}\left(\operatorname{proj}_{p}^{1}\right)=\operatorname{proj}_{p}^{1}(\mathbb{Z})=\mathbb{F}_{p}[\mathbb{Z}] / I^{p+1}$ is a $p+1$-dimensional $\mathbb{F}_{p^{\text {-vector }}}$ space. We know already that $\operatorname{proj}_{p-1}^{1}$ is a quotient of $\operatorname{proj}_{p}^{1}$ and that this is a semisimple functor with $p-1$ direct factors. So, every direct summand of the semisimple functor $p r o j_{p-1}^{1}$ is a direct factor of the head of $\operatorname{proj}_{p}^{1}$. Denote by $S_{0}, S_{1}, S_{2} \cdots S_{p-1}$ the simple direct factors of $\operatorname{proj}_{p-1}^{1}$ and let $S_{i}$ be of degree $i$. 
Then, since the degree 0 functors split off in any case, $P_{S_{0}}=S_{0}$. Moreover, $S_{0} \oplus$ $P_{S_{0}} \oplus P_{S_{1}} \oplus \cdots \oplus P_{S_{p-1}}$ is a direct factor of $\operatorname{proj}_{p}^{1}$.

We need to study the functors $P_{S_{i}}$. If all the composition factors of $P_{S_{i}}$ for an $i \leq p-1$ are of degree $p-1$ at most, then by Lemma 3.6 we get that $P_{S_{i}}$ is of degree at most $p-1$ as well. Since the category $\mathcal{A}_{\mathbb{F}_{p}}^{p-1}$ is semisimple, we get $P_{S_{i}}=S_{i}$.

Suppose that a degree $p$ simple polynomial functor $S$ is a composition factor of $P_{S_{i_{0}}}$. Then, since $\mathcal{A}_{\mathbb{F}_{p}}^{p-1}$ is semisimple, again by Lemma 3.6. $\operatorname{Ext}_{\mathcal{A}_{\mathbb{F}_{p}}^{p}}^{1}\left(S_{i_{0}}, S\right) \neq 0$. Now, simple functors are self-dual under the duality $(D F)(V):=F\left(V^{*}\right)^{*}$ (cf. [23] for functors in $\mathcal{F}_{\mathbb{F}_{p}}$ and Lemma 3.10 for simple functors in $\left.\mathcal{A}_{\mathbb{F}_{p}}\right)$. So, $\operatorname{Ext}_{\mathcal{A}_{\mathbb{F}_{p}}^{p}}^{1}\left(S, S_{i_{0}}\right)$ $\neq 0$. Since $\operatorname{Hom}_{\mathcal{A}_{\mathbb{F}_{p}}^{p}}\left(\operatorname{proj}_{p}^{1}, \mathbb{F}_{p} \otimes_{\mathbb{Z}}-\right)$ is a one-dimensional $\mathbb{F}_{p}$-vector space, this happens for precisely one $i_{0} \in\{1,2, \ldots, p-1\}$. We already know by Corollary 5.3 that $i_{0}=1$ and $S$ is the simple module corresponding to the trivial representation of $\mathbb{F}_{p} \mathfrak{S}_{p}$. This proves the claim.

Claim 5.13. For the projective cover $P_{L}$ of $L$ we get $\operatorname{rad}\left(P_{L}\right) / \operatorname{rad}^{2}\left(P_{L}\right) \simeq$ $\left(\mathbb{F}_{p} \otimes i d\right) \oplus L_{2}$, where $L_{2}$ is simple of degree $p, L_{2} \neq L$ and $\operatorname{rad}^{2}\left(P_{L}\right) \simeq L$ as well as $\operatorname{rad}^{3}\left(P_{L}\right)=0$.

Remark 5.14. We do not claim here that $\operatorname{soc}\left(P_{L}\right)$ is simple. However, the radical layer structure of $P_{L}$ can be described by

$$
\left(\mathbb{F}_{p} \otimes i d\right) \stackrel{L}{L} L_{2},
$$

where it is not clear if $\operatorname{soc}\left(P_{L}\right)$ is simple and isomorphic to $L$ or if the socle is isomorphic to $L \oplus L_{2}$ or to $L \oplus \mathbb{F}_{p} \otimes i d$. But $\operatorname{Hom}_{\mathcal{A}_{\mathbb{F}_{p}}^{p}}\left(\mathbb{F}_{p} \otimes i d^{\otimes p}, P_{L}\right)$ is the projective cover of the trivial $\mathbb{F}_{p} \mathfrak{S}_{p}$-module. This projective cover is uniserial with composition series

$$
\begin{gathered}
L \\
L_{2} \\
L
\end{gathered} .
$$

Since $L_{2}$ is simple of degree $p$, its image in $\mathbb{F}_{p} \mathfrak{S}_{p}$ is given by the known module structure of $\mathbb{F}_{p} \mathfrak{S}_{p}$. In particular, $L \neq L_{2}$. This shows that the only uniserial module of length 3 which is a quotient of $P_{L}$, if there is any, can have composition series

$$
\begin{gathered}
L \\
L_{2} \\
L
\end{gathered} .
$$

In particular, $\operatorname{soc}\left(P_{L}\right) \not 千 L \oplus L_{2}$.

Proof of Claim [5.13, By Corollary 5.3 we know that $\left(\mathbb{F}_{p} \otimes i d\right)$ is a composition factor of $\operatorname{top}\left(\operatorname{rad}\left(P_{L}\right)\right)$, and since $\operatorname{Hom}_{\mathcal{A}_{\mathbb{F}_{p}}^{p}}\left(\operatorname{proj}_{p}^{1}, \mathbb{F}_{p} \otimes_{\mathbb{Z}}-\right)$ is a one-dimensional $\mathbb{F}_{p^{-}}$ vector space we know that it has multiplicity 1 . Since the image of $P_{L}$ in $\mathbb{F}_{p} \mathfrak{S}_{p}-\bmod$ is uniserial with top and socle $L$ and simple $\operatorname{rad}\left(P_{L}\right) / \operatorname{soc}\left(P_{L}\right) \simeq L_{2}$, we have the above structure.

Claim 5.15. The projective cover $M=P_{\mathbb{F}_{p} \otimes i d}$ of $\mathbb{F}_{p} \otimes_{\mathbb{Z}}$ id has $\operatorname{rad}(M) / \operatorname{rad}^{2}(M) \simeq$ $L$. 
Proof. By Corollary 5.3 we know that $L$ is a direct factor of $\operatorname{top}(\operatorname{rad}(M))$. Since $\mathcal{A}_{\mathbb{F}_{p}}^{p-1}$ is semisimple, using Lemma 3.6 we see that no simple functor of degree $p-1$ at most can be a direct factor of $t o p(\operatorname{rad}(M))$. Suppose $t o p(\operatorname{rad}(M))$ has a second simple direct factor $T$ of degree $p$. Then, $\operatorname{Ext}_{\mathcal{A}_{\mathbb{F}_{p}}^{p}}^{1}\left(\mathbb{F}_{p} \otimes_{\mathbb{Z}} i d, T\right) \neq 0$. Simple functors are self-dual (cf. as above [23] for functors in $\mathcal{F}_{\mathbb{F}_{p}}$ and Lemma 3.10 for simple functors in $\left.\mathcal{A}_{\mathbb{F}_{p}}\right)$. So, $\operatorname{Ext}_{\mathcal{A}_{\mathbb{F}_{p}}^{p}}^{1}\left(T, \mathbb{F}_{p} \otimes_{\mathbb{Z}} i d\right) \neq 0$. But, we have seen in Corollary 5.3 that there is one simple functor of degree $p$ with a non-trivial extension group with $\mathbb{F}_{p} \otimes_{\mathbb{Z}} i d$, namely $L$. Moreover, since $\operatorname{proj}_{p}^{1}$ contains the projective cover of the simple degree 1-functor as a direct factor (see Claim 5.12), and since by Lemma 5.11 this is the only degree where non-trivial first extension groups can occur, we see that $T \simeq L$.

Suppose $L \oplus L \mid \operatorname{rad}(M)$. Then, $\operatorname{Ext}_{\mathcal{A}_{\mathbb{F}_{p}}^{p}}^{1}\left(\mathbb{F}_{p} \otimes_{\mathbb{Z}} i d, L\right)$ is two-dimensional at least, and again by the self-duality of the simple functors, $E x t_{\mathcal{A}_{\mathbb{F}_{p}}^{p}}^{1}\left(L, \mathbb{F}_{p} \otimes_{\mathbb{Z}} i d\right)$ is at least two-dimensional. Therefore, $\mathbb{F}_{p} \otimes_{\mathbb{Z}} i d$ occurs twice in $\operatorname{top}\left(\operatorname{rad}\left(P_{L}\right)\right)$. Since $M$ is a direct factor of $\operatorname{proj}_{p}^{1}$, the space $\operatorname{Hom}_{\mathcal{A}_{\mathbb{F}_{p}}^{p}}\left(\operatorname{proj}_{p}^{1},\left(\mathbb{F}_{p} \otimes_{\mathbb{Z}} i d\right)^{\otimes^{p}}\right)$ would be twodimensional at least. This contradiction shows that $\operatorname{rad}(M)$ has simple top $L$. Hence, $\operatorname{top}(\operatorname{rad}(M)) \simeq L$.

Remark 5.16. The radical layer structure of $M$ is therefore given by

$$
\begin{gathered}
\left(\mathbb{F}_{p} \otimes i d\right) \\
L \\
\operatorname{rad}^{2}(M)
\end{gathered},
$$

and by Claim 5.13 we get $\operatorname{rad}^{3}\left(P_{L}\right)=0$, and therefore we obtain $\operatorname{rad}^{4}(M)=0$.

Claim 5.17. For the projective cover $M$ of $\mathbb{F}_{p} \otimes_{\mathbb{Z}}$ id we get that top $\left(\operatorname{rad}^{2}(M)\right) \simeq$ $\mathbb{F}_{p} \otimes_{\mathbb{Z}} i d$.

Proof. Degree $p$ functors can only have extensions with degree $p$-functors or degree 1-functors by Lemma 5.11. Moreover, the structure of $P_{L}$ implies that we get that top $\left(\operatorname{rad}^{2}(M)\right)$ is a direct summand of $\operatorname{top}\left(\operatorname{rad}\left(P_{L}\right)\right)$, whence it is isomorphic to either 0 , or to $L_{2}$ (which is defined in Claim 5.13), or to $\mathbb{F}_{p} \otimes_{\mathbb{Z}} i d$, or to $L_{2} \oplus\left(\mathbb{F}_{p} \otimes_{\mathbb{Z}} i d\right.$ ).

Suppose $L_{2} \oplus\left(\mathbb{F}_{p} \otimes_{\mathbb{Z}} i d\right) \simeq \operatorname{top}\left(\operatorname{rad}^{2}(M)\right)$. We shall use the fact that by Claim 5.13 we know the structure of $P_{L}$.

We get two possibilities for the projective resolution of $\mathbb{F}_{p} \otimes_{\mathbb{Z}} i d$. Either

$$
P_{L} \hookrightarrow M \longrightarrow\left(\mathbb{F}_{p} \otimes_{\mathbb{Z}} i d\right)
$$

is exact, or

$$
L \hookrightarrow P_{L} \longrightarrow M \longrightarrow\left(\mathbb{F}_{p} \otimes_{\mathbb{Z}} i d\right)
$$

is exact.

In the second case, $\operatorname{Ext}_{\mathcal{A}_{\mathbb{F}_{p}}^{p}}^{2}\left(\left(\mathbb{F}_{p} \otimes_{\mathbb{Z}} i d\right), L\right) \neq 0$. By the self-duality of the simple functors, $\operatorname{Ext}_{\mathcal{A}_{\mathbb{F}_{p}}^{p}}^{2}\left(L,\left(\mathbb{F}_{p} \otimes_{\mathbb{Z}} i d\right)\right) \neq 0$. Our information is sufficient for being able to write down the first terms of the projective resolution of $L$,

$$
0 \longleftarrow L \longleftarrow P_{L} \longleftarrow M \oplus P_{L_{2}} \longleftarrow P_{L} \oplus P_{L_{3}} \longleftarrow \ldots
$$

for some projective $P_{L_{3}}$, for some simple object $L_{3}$ of degree $p$, given by the known projective resolution of the trivial $\mathbb{F}_{p} \mathfrak{S}_{p}$-module. Since $p \neq 2$, we get $L_{3} \nsucceq L$. In 
any case $\operatorname{Hom}\left(P_{L} \oplus P_{L_{3}},\left(\mathbb{F}_{p} \otimes_{\mathbb{Z}} i d\right)\right)=0$, and therefore $\operatorname{Ext}_{\mathcal{A}_{\mathbb{F}_{p}}^{p}}^{2}\left(\left(\mathbb{F}_{p} \otimes_{\mathbb{Z}} i d\right), L\right)=0$. This contradiction excludes that the case

$$
L \hookrightarrow P_{L} \longrightarrow M \longrightarrow\left(\mathbb{F}_{p} \otimes_{\mathbb{Z}} i d\right)
$$

is exact.

If $P_{L} \hookrightarrow M \longrightarrow\left(\mathbb{F}_{p} \otimes_{\mathbb{Z}} i d\right)$ is exact, the projective dimension of $\left(\mathbb{F}_{p} \otimes_{\mathbb{Z}} i d\right)$ is 1 . But, we know by Theorem 2.6 and the example following it, that $\operatorname{Ext}_{\mathcal{F}_{\mathbb{F}_{p}}^{p}}^{2}(i d, i d) \neq 0$. By consequence, also $E x t_{\mathcal{A}_{\mathbb{F}_{p}}^{p}}^{2}\left(\left(\mathbb{F}_{p} \otimes_{\mathbb{Z}} i d\right),\left(\mathbb{F}_{p} \otimes_{\mathbb{Z}} i d\right)\right) \neq 0$, and therefore the projective dimension of $\left(\mathbb{F}_{p} \otimes_{\mathbb{Z}} i d\right)$ is at least 2 .

These two observations exclude $L_{2} \oplus\left(\mathbb{F}_{p} \otimes_{\mathbb{Z}} i d\right) \simeq \operatorname{top}\left(\operatorname{rad}^{2}(M)\right)$.

Suppose $L_{2} \simeq \operatorname{top}\left(\operatorname{rad}^{2}(M)\right)$. Then, using the structure of $P_{L}$, we get either $\operatorname{rad}^{3}(M)=0$ or $\operatorname{rad}^{3}(M)=L$, and then $\operatorname{rad}^{4}(M)=0$.

If $\operatorname{rad}^{3}(M)=0$, then we get a non-split exact sequence

$$
0 \longleftarrow\left(\mathbb{F}_{p} \otimes_{\mathbb{Z}} i d\right) \longleftarrow M \longleftarrow P_{L} \longleftarrow M \longleftarrow L_{2} \longleftarrow 0,
$$

and therefore $\operatorname{Ext}_{\mathcal{F}_{\mathbb{F}_{p}}^{p}}^{3}\left(L_{2},\left(\mathbb{F}_{p} \otimes_{\mathbb{Z}} i d\right)\right) \neq 0$. Dualizing, $\operatorname{Ext}_{\mathcal{F}_{\mathbb{F}_{p}}^{p}}^{3}\left(\left(\mathbb{F}_{p} \otimes_{\mathbb{Z}} i d\right), L_{2}\right) \neq 0$. Our knowledge of the various projective covers of simples is sufficient to write down the first terms of the projective resolution of $L_{2}$. We get

$$
0 \longleftarrow L_{2} \longleftarrow P_{L_{2}} \longleftarrow P_{L} \oplus P_{L_{3}} \longleftarrow M \oplus P_{L_{2}} \oplus P_{L_{4}} \longleftarrow P_{L} \oplus P_{L_{3}} \oplus P_{L_{5}} \longleftarrow \ldots
$$

for projective objects $P_{L_{4}}$ and $P_{L_{5}}$ corresponding to degree $p$ simple functors $L_{4}$ and $L_{5}$, given by the known projective resolution of the trivial $\mathbb{F}_{p} \mathfrak{S}_{p}$-module. Moreover, $L_{3}$ and $L_{5}$ are both different from $L_{2}$, since $p \geq 5$. This implies $E x t_{\mathcal{F}_{\mathbb{F}_{p}}^{p}}^{3}\left(\left(\mathbb{F}_{p} \otimes_{\mathbb{Z}} i d\right), L_{2}\right)=0$. This contradiction excludes this case as well.

So, assume $\operatorname{rad}^{3}(M)=L$ and $\operatorname{rad}^{4}(M)=0$. This is impossible since then the endomorphism ring of $M$ would be one-dimensional. This contradicts the fact that $\operatorname{End}_{\mathcal{A}_{\mathbb{F}_{p}}^{p}}\left(\operatorname{proj}_{p}^{1}\right)$ is $p+1$-dimensional.

Hence, $L_{2} \not$ top $\left(\operatorname{rad}^{2}(M)\right)$.

We still have the possibility that $\operatorname{rad}^{2}(M)=0$. But again, this would imply that $\operatorname{End}_{\mathcal{A}_{\mathbb{F}_{p}}^{p}}(M)$ would be one-dimensional and therefore $\operatorname{End}_{\mathcal{A}_{\mathbb{F}_{p}}^{p}}\left(p r o j_{p}^{1}\right)$ is p-dimensional. A contradiction.

This proves the claim.

Claim 5.18. $\operatorname{rad}^{3}(M)=0$.

Proof. We know by Claim 5.13 that $\operatorname{rad}^{3}\left(P_{L}\right)=0$. Since by Claim 5.15 we have $\operatorname{top}(\operatorname{rad}(M)) \simeq L$, one sees that $\operatorname{rad}^{4}(M)=0$. Moreover, $\operatorname{rad}^{3}(M)$ is either 0 or $L$, since $\operatorname{top}\left(\operatorname{rad}^{2}(M)\right) \simeq\left(\mathbb{F}_{p} \otimes_{\mathbb{Z}} i d\right)$ by Claim 5.17 and $\operatorname{top}(\operatorname{rad}(M)) \simeq L$ by Claim 5.15

Suppose $\operatorname{rad}^{3}(M) \simeq L$. Then $M$ is uniserial with composition length 4 , and $\operatorname{top}(\operatorname{rad}(M))=L$. Therefore $P_{L}$ maps onto $\operatorname{rad}(M)$ with its image being a uniserial module $N$ of length 3 with $\operatorname{rad}(N) / \operatorname{soc}(N)=\mathbb{F}_{p} \otimes i d$.

But this contradicts the structure of $P_{L}$ as described in Claim 5.13 and in particular Remark 5.14

This proves the claim.

Examining what we have shown implies that $\operatorname{proj}_{p}^{1}$ is as stated in Proposition 5.9 
We now come to our first main result in describing the structure of $\Gamma_{\mathbb{F}_{p}, 0}$. For the relevant definitions on Brauer tree algebras we refer to Section 4.

Theorem 5.19. $\Gamma_{\mathbb{F}_{p}, 0}$ is a Brauer tree algebra over $\mathbb{F}_{p}$ without exceptional vertex and associated to a stem with $p$ edges:

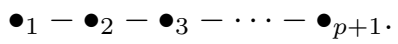

Proof. The case $p \leq 3$ is a consequence of Drozd's results. By Proposition [5.9 we know that the projective cover of the functor $\mathbb{F}_{p} \otimes_{\mathbb{Z}}-$ is uniserial with top and socle $\mathbb{F}_{p} \otimes_{\mathbb{Z}}-$ and with second layer $V$, where $V$ is the simple functor corresponding to the trivial $\mathbb{F}_{p} \mathfrak{S}_{p}$-module.

We know furthermore that except for the projective cover of $\mathbb{F}_{p} \otimes_{\mathbb{Z}}$ - only the projective indecomposable functor $P_{L}$ has a composition factor $\mathbb{F}_{p} \otimes_{\mathbb{Z}}-$ and that this composition factor is a direct summand of $\operatorname{top}\left(\operatorname{rad}\left(P_{L}\right)\right)$.

Since we know that the principal block of $\mathbb{F}_{p} \mathfrak{S}_{p}$ is a Brauer tree algebra without exceptional vertex associated to a stem with $p$ vertices, this means that we only need to show that $\mathbb{F}_{p} \otimes_{\mathbb{Z}}$ - is not in the socle of $P_{L}$, since the only basic algebra with the composition series as a Brauer tree algebra associated to a stem is actually a Brauer tree algebra associated to a stem.

For this we use the duality $D$ on the category of polynomial functors. The projective indecomposable functor $P_{L}$ is a direct factor of $\mathbb{F}_{p} \otimes_{\mathbb{Z}} \underbrace{i d \otimes_{\mathbb{Z}} \cdots \otimes_{\mathbb{Z}} i d}_{p \text { factors }}$,

since this is the projective cover of all the degree $p$ simple functors.

It is clear that $\mathbb{F}_{p} \otimes_{\mathbb{Z}} \underbrace{i d \otimes_{\mathbb{Z}} \cdots \otimes_{\mathbb{Z}} i d}_{p \text { factors }}$ is self-dual. Since all the simple functors are self-dual, also $D P_{L} \simeq P_{L}$. If $\mathbb{F}_{p} \otimes_{\mathbb{Z}}-$ is in the socle of $P_{L}$, the simple functor $D\left(\mathbb{F}_{p} \otimes_{\mathbb{Z}}-\right) \simeq \mathbb{F}_{p} \otimes_{\mathbb{Z}}-$ is in the top of $D P_{L} \simeq P_{L}$, but the top of $P_{L}$ is $L$ by definition.

This proves the theorem.

\section{LifTING TO CHARACTERISTIC 0}

\subsection{Lifting Brauer tree algebras to orders.}

Proposition 6.1. Let $R$ be a complete discrete valuation ring with residue field $k$ and field of fractions $K$. Let $B$ be a Brauer tree algebra over $k$ associated to a Brauer tree which is a stem without exceptional vertex. Let $\Lambda$ be an $R$-order. Then, for any proper two-sided ideal $I \neq 0$ of $B$ we get that

$$
\Lambda \otimes_{R} k \simeq B / I \Longrightarrow \operatorname{rank}_{\mathbb{Z}}\left(K_{0}\left(K \otimes_{R} \Lambda\right)\right) \leq \operatorname{rank}_{\mathbb{Z}}\left(K_{0}(B / I)\right) .
$$

Proof. We shall first suppose that $\Lambda$ is indecomposable and that $I \leq \operatorname{rad}(B)$.

Let $S_{1}, S_{2}, \ldots, S_{n}$ be representatives of the simple $B$-modules. The projective cover $P_{i}$ of $S_{i}$ then has a composition series where $\operatorname{soc}\left(P_{i}\right) \simeq S_{i}$ and

$$
\operatorname{rad}\left(P_{i}\right) / \operatorname{soc}\left(P_{i}\right) \simeq S_{i-1} \oplus S_{i+1}
$$

for all $i \in\{2,3 \ldots, n-1\}$,

$$
\operatorname{rad}\left(P_{1}\right) / \operatorname{soc}\left(P_{1}\right) \simeq S_{2}
$$

and

$$
\operatorname{rad}\left(P_{n}\right) / \operatorname{soc}\left(P_{n}\right) \simeq S_{n-1} .
$$


Denote $\bar{B}:=B / I$. Since $I \leq \operatorname{rad}(B)$, we get that $\bar{B}$ has the same number of simple modules, and moreover, the simple $\bar{B}$-modules and the simple $B$-modules coincide by the epimorphism $B \longrightarrow \bar{B}$. Therefore the projective indecomposable $\bar{B}$-modules are $\bar{P}_{i}:=\bar{B} \otimes_{B} P_{i}$ for $i \in\{1,2, \ldots, n\}$. Moreover, $\bar{P}_{i}$ is the projective cover of $S_{i}$ as a $\bar{B}$-module.

Extending $k$ if necessary, we may assume that the field of fractions $K$ of $R$ is a splitting field for $\Lambda$, since extending $K$ does not decrease the rank of the Grothendieck group, using the Noether-Deuring theorem. Since $k$ is a splitting field for $B$ and for $B / I$, and since $k \otimes_{R} \Lambda \simeq \bar{B}$, the Cartan matrix of $\bar{B}$ is symmetric (cf. e.g. [21, Proposition 4.2.11]). Since the Cartan matrix of $B$ equals

$$
C:=\left(\begin{array}{cccccc}
2 & 1 & 0 & \ldots & \ldots & 0 \\
1 & 2 & 1 & \ddots & & \vdots \\
0 & 1 & \ddots & \ddots & \ddots & \vdots \\
\vdots & \ddots & \ddots & \ddots & 1 & 0 \\
\vdots & & \ddots & 1 & 2 & 1 \\
0 & \ldots & \ldots & 0 & 1 & 2
\end{array}\right)
$$

we see that the composition length of $\bar{P}_{i}$ differs from the composition length of $P_{i}$ by at most 1 . Indeed, if this would not be the case, then the composition matrix of $\bar{B}$ would be decomposable into at least two blocks and $\bar{B}$ would be decomposable as an algebra. But, since $\Lambda$ is indecomposable, so is $k \otimes_{R} \Lambda \simeq \bar{B}$. So, $I \leq \operatorname{soc}(B)$.

Since $R$ is complete, we may assume that $B$ and $\Lambda$ are both basic algebras.

Let $Q_{i}$ be the projective cover of $\bar{P}_{i}$ as a $\Lambda$-module. Hence, $k \otimes_{R} Q_{i} \simeq \bar{P}_{i}$. Since

$$
\operatorname{dim}_{K} \operatorname{Hom}_{K \Lambda}\left(K Q_{i}, K Q_{j}\right)=\operatorname{dim}_{R} \operatorname{Hom}_{\Lambda}\left(Q_{i}, Q_{j}\right)=\operatorname{dim}_{k} \operatorname{Hom}_{\bar{B}}\left(\bar{P}_{i}, \bar{P}_{j}\right)
$$

we know that $K Q_{i}$ and $K Q_{j}$ do not have a character in common if $|i-j|>1$ and do have one character in common if $|i-j|=1$. Since $\operatorname{dim}_{k} \operatorname{Hom}_{\bar{B}}\left(\bar{P}_{i}, \bar{P}_{i}\right) \in\{1,2\}$, the character of $K Q_{i}$ for all $i \in\{1, \ldots, n\}$ is a sum of at most two irreducible characters, and in the case of two characters these are non-isomorphic. Now, since $\operatorname{Hom}_{K \Lambda}\left(K Q_{i}, K Q_{j}\right)=0$ if $|i-j|>1$, it follows that if

$$
\operatorname{dim}_{K} \operatorname{Hom}_{K \Lambda}\left(K Q_{i_{0}}, K Q_{i_{0}}\right)=1,
$$

then $i_{0} \in\{1, n\}$. Otherwise, the character of $K Q_{i_{0}}$ would be a constituent of $K Q_{i_{0}+1}$, of $K Q_{i_{0}}$ and of $K Q_{i_{0}-1}$, which then implies $H$ om $_{K \Lambda}\left(K Q_{i_{0}+1}, K Q_{i_{0}-1}\right) \neq$ 0 . This would give a contradiction. This gives that $I$ equals either $S_{1}$ or $S_{n}$ or $S_{1} \oplus S_{n}$.

Suppose now that $S_{1}$ is a direct factor of $I$ (as a left module) and suppose

$$
\operatorname{rank}_{\mathbb{Z}}\left(K_{0}\left(K \otimes_{R} \Lambda\right)\right)>\operatorname{rank}_{\mathbb{Z}}\left(K_{0}(B)\right) .
$$

We shall prove that $S_{1}$ is not a direct factor of $I$. By symmetry, then neither is $S_{n}$ a direct factor of $I$, and therefore, $I=0$.

Under these hypotheses, $\operatorname{dim}\left(\bar{P}_{1}\right)=2$, and as a consequence also $\operatorname{dim}_{R}\left(Q_{1}\right)=2$. So, for the Wedderburn components corresponding to $K \otimes_{R} Q_{1}$ in $K \otimes_{R} \Lambda$ we have two possibilities. Either $K \otimes_{R} Q_{1}$ is a sum of two one-dimensional characters or $K \otimes_{R} Q_{1}$ is isomorphic to one two-dimensional character. Since $K \otimes_{R} \Lambda$ admits at least $n+1$ irreducible characters, $K \otimes_{R} Q_{1}$ must have two constituents. So, $K \otimes_{R} Q_{1}$ is a sum of two one-dimensional characters. 
But now, let $\left\{e_{i} \mid i \in\{1, \ldots, n\}\right\}$ be an orthogonal set of primitive idempotents with $\Lambda e_{i} \simeq Q_{i}$. Then, since $e_{1}$ and $e_{2}$ must be non-zero on the common Wedderburn component of $K Q_{1}$ and $K Q_{2}$, we get $e_{2} e_{1} \neq 0$. This is a contradiction to the fact that $e_{1}$ and $e_{2}$ are orthogonal.

We have to deal with the case of $\Lambda$ being decomposable. The structure of $B$ implies that in this case, $B / I$ is a direct product of algebras we have dealt with in the earlier case, and copies of $k$. By induction on the number of simple modules of each indecomposable factor the result holds for each of the pieces as well. Summing up for all of these pieces, we get the desired result.

Finally, we have to deal with the case that $I$ is not contained in $\operatorname{rad}(B)$. The same argument as for $\Lambda$ decomposable applies here as well.

This proves the proposition.

6.2. Proving that the Baues-Dreckmann-Franjou-Pirashvili ring is an order. Since $\hat{\mathbb{Z}}_{p}$ is a complete discrete valuation ring, we may lift idempotents from $\Gamma_{\mathbb{F}_{p}}^{p}$ to $\Gamma_{\hat{\mathbb{Z}}_{p}}^{p}$. Hence, there is an indecomposable direct factor $\Gamma_{\hat{\mathbb{Z}}_{p}, 0}^{p}$ of the rank one free module $\Gamma_{\hat{\mathbb{Z}}_{p}}^{p}$ which maps surjectively to $\Gamma_{\mathbb{F}_{p}, 0}^{p}$. Let $T_{0}^{p}:=t\left(\Gamma_{\hat{\mathbb{Z}}_{p}, 0}^{p}\right)$ be the torsion ideal in $\Gamma_{\hat{\mathbb{Z}}_{p}, 0}^{p}$ and define $\Lambda_{\hat{\mathbb{Z}}_{p}, 0}^{p}:=\Gamma_{\hat{\mathbb{Z}}_{p}, 0}^{p} / T_{0}^{p}$.

Proposition 6.2. $\Lambda_{\hat{\mathbb{Z}}_{p}, 0}^{p}$ is an order. Moreover, $\hat{\mathbb{Q}}_{p} \otimes_{\hat{\mathbb{Z}}_{p}} \Lambda_{\hat{\mathbb{Z}}_{p}, 0}^{p}$ is a direct product of $p+1$ matrix rings over $\hat{\mathbb{Q}}_{p}$ and up to isomorphism there are at most $p$ simple $\Lambda_{\hat{\mathbb{Z}}_{p}, 0}^{p}$-modules.

Proof. In fact, $\hat{\mathbb{Q}}_{p} \otimes_{\hat{\mathbb{Z}}_{p}} \Lambda_{\hat{\mathbb{Z}}_{p}}^{p}=\hat{\mathbb{Q}}_{p} \otimes_{\hat{\mathbb{Z}}_{p}} \Gamma_{\hat{\mathbb{Z}}_{p}}^{p}$ and their common module categories are equivalent to the category of polynomial functors $\mathbb{Z}-\bmod \longrightarrow \hat{Q}_{p}-\bmod$ of degree at most $p$ by Lemma 1.3

By Friedlander-Suslin [16, the category of precisely degree $n$ polynomial functors $\mathbb{Z}-\bmod \longrightarrow \hat{Q}_{p}-\bmod$ is equivalent to the category of strict polynomial functors $\hat{Q}_{p}-\bmod \longrightarrow \hat{Q}_{p}-\bmod$ of degree $n$, and this category is equivalent to the category of modules over the Schur algebra $S_{\hat{\mathbb{Q}}_{p}}(n, n)$. Moreover, the category of strict polynomial functors of degree at most $n$ is equivalent to the direct sum of the category of strict polynomial functors of exact degree $m$ for each $m \in\{0,1, \ldots, n\}$. The Schur algebra $S_{\hat{\mathbb{Q}}_{p}}(p, p)$ is split semisimple (cf. Green [17]) with exactly $p+1$ simple modules. This shows that $\hat{\mathbb{Q}}_{p} \otimes_{\hat{\mathbb{Z}}_{p}} \Lambda_{\hat{\mathbb{Z}}_{p}, 0}^{p}$ is a direct product of $p+1$ full matrix rings over $\hat{\mathbb{Q}}_{p}$. Moreover, this shows also that $\Lambda_{\hat{\mathbb{Z}}_{p}}^{p}$ is an order since it is by definition torsion free and contains a basis of the semisimple algebra $\hat{\mathbb{Q}}_{p} \otimes_{\hat{\mathbb{Z}}_{p}} \Lambda_{\hat{\mathbb{Z}}_{p}}^{p}$ (which is Morita equivalent to $\prod_{i=0}^{p} S_{\hat{\mathbb{Q}}_{p}}(i, i)$ ).

In order to prove the second statement we just observe that the number of simple objects in $\mathcal{A}_{\mathbb{F}_{p}}^{p}$ equals the number of simple objects in $\mathcal{F}_{\mathbb{F}_{p}}^{p}$ by Lemma 3.10 . Moreover, since $\Lambda_{\hat{\mathbb{Z}}_{p}, 0}^{p}$ is a quotient of $\Gamma_{\hat{\mathbb{Z}}_{p}, 0}^{p}$, every simple $\Lambda_{\hat{\mathbb{Z}}_{p}, 0}^{p}$-module induces a simple $\Gamma_{\hat{\mathbb{Z}}_{p}, 0}^{p}$-module. We know that $\Gamma_{\mathbb{F}_{p}, 0}^{p}$ is a Brauer tree algebra with $p$ simple modules. Moreover, $\mathbb{F}_{p} \otimes_{\hat{\mathbb{Z}}_{p}} \Gamma_{\hat{\mathbb{Z}}_{p}, 0}^{p} \simeq \Gamma_{\mathbb{F}_{p}, 0}^{p}$, and so $\Gamma_{\hat{\mathbb{Z}}_{p}, 0}^{p}$ admits $p$ simple modules. As a consequence $\Lambda_{\hat{\mathbb{Z}}_{p}, 0}^{p}$ admits at most $p$ simple modules. This proves the proposition. 
Proposition 6.3. $t\left(\Gamma_{\mathbb{F}_{p}, 0}^{p}\right)=0$ and therefore $\Lambda_{\hat{\mathbb{Z}}_{p}, 0}^{p}=\Gamma_{\hat{\mathbb{Z}}_{p}, 0}^{p}$.

Proof. This is a consequence of Proposition 6.2. Theorem 5.19 and Proposition 6.1. Indeed, since $\mathbb{F}_{p} \otimes_{\hat{\mathbb{Z}}_{p}}-$ is right exact, the epimorphism

$$
\Gamma_{\hat{\mathbb{Z}}_{p}, 0}^{p} \longrightarrow \Lambda_{\hat{\mathbb{Z}}_{p}, 0}^{p}
$$

induces an epimorphism

$$
B=\Gamma_{\mathbb{F}_{p}, 0}^{p} \longrightarrow \Lambda_{\hat{\mathbb{Z}}_{p}, 0}^{p} \otimes_{\hat{\mathbb{Z}}_{p}} \mathbb{F}_{p}
$$

with kernel $I$, for $B$ being a Brauer tree algebra associated to a stem with $p$ edges and without exceptional vertex (Theorem [5.19). Since $t\left(\Gamma_{\hat{\mathbb{Z}}_{p}, 0}^{p}\right) \subseteq \operatorname{rad}\left(\Gamma_{\mathbb{F}_{p}, 0}^{p}\right)$ by Proposition 6.2. $I \leq \operatorname{rad}(B)$. Since $B / I \simeq \Lambda_{\hat{\mathbb{Z}}_{p}, 0}^{p} \otimes_{\hat{\mathbb{Z}}_{p}} \mathbb{F}_{p}$ for an order $\Lambda_{\hat{\mathbb{Z}}_{p}, 0}^{p}$, Proposition 6.1 implies that in this case $I=0$. Hence, $\mathbb{F}_{p} \otimes_{\hat{\mathbb{Z}}_{p}} t\left(\Gamma_{\hat{\mathbb{Z}}_{p}, 0}^{p}\right)=0$, and therefore $t\left(\Gamma_{\hat{\mathbb{Z}}_{p}, 0}^{p}\right)=0$. This proves the proposition.

6.3. Describing the order; the main result. We shall describe $\Lambda_{\hat{\mathbb{Z}}_{p}, 0}^{p}$ and prove our main result. For this purpose we introduce some notation (cf. [21, Section 4.4]). Let

and

$$
\hat{\mathbb{Z}}_{p} \frac{p^{i}}{\mathbb{Z}_{p}} \quad:=\left\{(a, b) \in \hat{\mathbb{Z}}_{p} \times \hat{\mathbb{Z}}_{p} \mid a-b \in p^{i} \hat{\mathbb{Z}}_{p}\right\}
$$

$$
\hat{\mathbb{Z}}_{p} \hat{\mathbb{Z}}_{p} \quad:=\hat{\mathbb{Z}}_{p} \frac{p}{p} \hat{\mathbb{Z}}_{p} .
$$

The following is the main result of our paper.

Theorem 6.4. Let $\mathcal{A}_{\hat{\mathbb{Z}}_{p}}^{p}$ be the category of polynomial functors from free abelian groups to $\hat{\mathbb{Z}}_{p}$-modules and of degree at most $p$. Then, $\mathcal{A}_{\hat{\mathbb{Z}}_{p}}^{p}$ is equivalent to $\Gamma_{\hat{\mathbb{Z}}_{p}}^{p}$-mod, where

$$
\Gamma_{\hat{\mathbb{Z}}_{p}}^{p}:=\left(\prod_{1<n<p} \hat{\mathbb{Z}}_{p}\right) \times\left(\prod_{\lambda \vdash p \text { and } \lambda \text { not a hook }} \hat{\mathbb{Z}}_{p}\right) \times \Lambda_{\hat{\mathbb{Z}}_{p}, 0}^{p}
$$

and where

$$
\begin{aligned}
& \Lambda_{\hat{\mathbb{Z}}_{p}, 0}^{p} \simeq \hat{\mathbb{Z}}_{p} \oplus\left(\begin{array}{ll}
\hat{\mathbb{Z}}_{p} & \hat{\mathbb{Z}}_{p} \\
(p) & \hat{\mathbb{Z}}_{p}
\end{array}\right) \oplus\left(\begin{array}{ll}
\hat{\mathbb{Z}}_{p} & \hat{\mathbb{Z}}_{p} \\
(p) & \hat{\mathbb{Z}}_{p}
\end{array}\right) \oplus \ldots \oplus\left(\begin{array}{cc}
\hat{\mathbb{Z}}_{p} & \hat{\mathbb{Z}}_{p} \\
(p) & \hat{\mathbb{Z}}_{p}
\end{array}\right) \oplus \hat{\mathbb{Z}}_{p} \\
& =\left\{\left(d_{0}\right) \times\left(\prod_{j=1}^{p-1}\left(\begin{array}{cc}
a_{j} & b_{j} \\
c_{j} & d_{j}
\end{array}\right)\right) \times\left(a_{p}\right)\left|\forall j: a_{j}, b_{j}, c_{j}, d_{j} \in \hat{\mathbb{Z}}_{p} ; p\right| c_{j} ; p \mid\left(d_{j}-a_{j-1}\right)\right\}
\end{aligned}
$$

is a Green order with $p$ isomorphism classes of indecomposable projective modules.

Remark 6.5. Roggenkamp described the orders $\Lambda$ which admit a set of lattices with periodic projective resolutions encoded by a Brauer tree ([34]; see also 21]). Roggenkamp called these orders Green orders and he described their structure in great detail.

Proof of the theorem. The case $p \leq 3$ was done by Drozd. Hence we may suppose that $p \geq 5$. Since $\mathbb{F}_{p} \otimes_{\hat{\mathbb{Z}}_{p}} \Lambda_{\hat{\mathbb{Z}}_{p}, 0}^{p}$ is a Brauer tree algebra, there is a set of $\mathbb{F}_{p} \otimes_{\hat{\mathbb{Z}}_{p}} \Lambda_{\hat{\mathbb{Z}}_{p}, 0}^{p}$ modules having a periodic projective resolution given by the Brauer tree of $\Lambda_{\mathbb{F}_{p}, 0}^{p}$. 
Lifting these projective resolutions to the order $\Lambda_{\hat{\mathbb{Z}}_{p}, 0}^{p}$ gives a periodic projective resolution of certain $\Lambda_{\hat{\mathbb{Z}}_{p}, 0}^{p}$-modules $M_{i}$. These periodic resolutions are encoded by the same Brauer tree. It remains to show that the modules $M_{i}$ are lattices. Actually, this is automatic. Indeed, since the resolution is periodic, each module $M_{i}$ is also a kernel of a differential, after a complete period of the periodic projective resolution. Hence, $\Lambda_{\hat{\mathbb{Z}}_{p}, 0}^{p}$ is a Green order with Brauer tree being a stem with $p$ edges and without exceptional vertex.

We have to show that the maximal overorder of the Green order $\Lambda_{\hat{\mathbb{Z}}_{p}, 0}^{p}$ is a direct product of matrix rings over $\hat{\mathbb{Z}}_{p}$ and that the image of $\Lambda_{\hat{\mathbb{Z}}_{p}, 0}^{p}$ in each of the matrix rings is a hereditary order.

The first part is clear since $\hat{\mathbb{Q}}_{p}$ is a splitting field of $\Lambda_{\hat{\mathbb{Z}}_{p}, 0}^{p}$, and $\Lambda_{\hat{\mathbb{Z}}_{p}, 0}^{p}$ can be embedded into a direct product of matrix rings over the ring of integers in $\hat{\mathbb{Q}}_{p}$ (see e.g. 33]). Let $e_{1}, e_{2}, \ldots, e_{p+1}$ be a complete set of primitive pairwise orthogonal idempotents of the center of $\hat{\mathbb{Q}}_{p} \otimes_{\hat{\mathbb{Z}}_{p}} \Lambda_{\hat{\mathbb{Z}}_{p}, 0}^{p}$. Then,

$$
\prod_{j=1}^{p-1}\left(\Lambda_{\hat{\mathbb{Z}}_{p}, 0}^{p} \cdot e_{i}\right) \simeq \hat{\mathbb{Z}}_{p} \times \prod_{j=1}^{p-1}\left(\begin{array}{cc}
\hat{\mathbb{Z}}_{p} & \hat{\mathbb{Z}}_{p} \\
\left(p^{x_{j}}\right) & \hat{\mathbb{Z}}_{p}
\end{array}\right) \times \hat{\mathbb{Z}}_{p}
$$

for some $x_{j} \in \mathbb{N} \backslash\{0\}$. Moreover, since $\Lambda_{\hat{\mathbb{Z}}_{p}, 0}^{p} \otimes_{\hat{\mathbb{Z}}_{p}} \mathbb{F}_{p}$ is a Brauer tree algebra without exceptional vertex, $x_{1}=x_{2}=\cdots=x_{p-1}$, and as a consequence, if one of the matrix rings is hereditary, all of them are hereditary. The structure theory of Green orders (cf. Roggenkamp [34]; see also [21, Section 4.4]) and of hereditary orders (cf. e.g. Reiner [33]) then gives the statement.

Define a functor

$$
\operatorname{Hom}_{\mathcal{A}_{\mathbb{\mathbb { Z }}_{p}}^{p}}\left(\hat{\mathbb{Z}}_{p} \otimes_{\mathbb{Z}}\left(\bigotimes_{j=1}^{p} i d\right),-\right): \mathcal{A}_{\hat{\mathbb{Z}}_{p}}^{p} \longrightarrow \hat{\mathbb{Z}}_{p} \mathfrak{S}_{p}-\bmod
$$

where we again use that the functor $\hat{\mathbb{Z}}_{p} \otimes_{\mathbb{Z}}\left(\bigotimes_{j=1}^{p} i d\right): \mathbb{Z}-$ free $\longrightarrow \hat{\mathbb{Z}}_{p}-\bmod$ carries a natural $\hat{\mathbb{Z}}_{p}$-linear $\mathfrak{S}_{p}$ action. Denote for notational simplicity $E:=$ $\operatorname{Hom}_{\mathcal{A}_{\mathbb{\mathbb { Z }}_{p}}^{p}}\left(\hat{\mathbb{Z}}_{p} \otimes_{\mathbb{Z}}\left(\bigotimes_{j=1}^{p} i d\right),-\right)$. By Lemma 3.7 this functor is just the $p-1$-th cross effect, and by Lemma 3.6 this functor $E$ is exact.

Since $E$ is exact, and since by definition $E$ is represented by $P:=\hat{\mathbb{Z}}_{p} \otimes_{\mathbb{Z}}$ $\left(\bigotimes_{j=1}^{p} i d\right)$, this object $P$ is projective. Let $e$ be an idempotent in $\Gamma_{\hat{\mathbb{Z}}_{p}}^{p}$ which corresponds to the projective indecomposable $\Gamma_{\hat{\mathbb{Z}}_{p}}^{p}$-modules which occur in $P$. Then, replacing $\mathcal{A}_{\hat{\mathbb{Z}}_{p}}^{p}$ by $\Gamma_{\hat{\mathbb{Z}}_{p}}^{p}-\bmod$ the functor $\operatorname{Hom}_{\mathcal{A}_{\hat{\mathbb{Z}}_{p}}^{p}}\left(\hat{\mathbb{Z}}_{p} \otimes_{\mathbb{Z}}\left(\bigotimes_{j=1}^{p} i d\right),-\right)$ becomes the functor $E: \Gamma_{\hat{\mathbb{Z}}_{p}}^{p}-\bmod \longrightarrow \hat{\mathbb{Z}}_{p} \mathfrak{S}_{p}-\bmod$ and $E$ is just multiplication by $e$.

We need to show that $\operatorname{End}_{\mathcal{A}_{\widehat{\mathbb{z}}_{p}}}(P) \simeq \hat{\mathbb{Z}}_{p} \mathfrak{S}_{p}$, where the action is given by permutation of components in the tensor product. Once this is done, we know that $\operatorname{End}_{\mathcal{A}_{\hat{\mathbb{Z}}_{p}}}(P) \simeq e \cdot \Gamma_{\hat{\mathbb{Z}}_{p}}^{p} \cdot e \simeq \hat{\mathbb{Z}}_{p} \mathfrak{S}_{p}$ and we observe that for all idempotents in $\Gamma_{\hat{\mathbb{Z}}_{p}}^{p}$ we get that this product $e \cdot \Gamma_{\hat{\mathbb{Z}}_{p}}^{p} \cdot e$ is again a product of Green orders with the same order of congruences. Since $\hat{\mathbb{Z}}_{p} \mathfrak{S}_{p}$ is a Green order with congruences modulo $p$ only, we get that $x=1$. 
Claim 6.6. $\operatorname{End}_{\mathcal{A}_{\hat{\mathbb{Z}}_{p}}}\left(\hat{\mathbb{Z}}_{p} \otimes_{\mathbb{Z}}\left(\bigotimes_{j=1}^{p} i d\right)\right) \simeq \hat{\mathbb{Z}}_{p} \mathfrak{S}_{p}$.

Proof. The proof given by Piriou-Schwartz [31, Lemma 1.9] of the corresponding statement for $\mathcal{F}_{\mathbb{F}_{p}}$ carries over literally. For the reader's convenience we recall the (short) arguments.

Given an $x=\sum_{\sigma \in \mathfrak{S}_{p}} x_{\sigma} \sigma \in \hat{\mathbb{Z}}_{p} \mathfrak{S}_{p}$, associate to this $x$ the natural transformation $\eta_{x}$ in $\operatorname{End}_{\mathcal{A}_{\hat{\mathbb{Z}}_{p}}}\left(\otimes_{j=1}^{p}\left(\hat{\mathbb{Z}}_{p} \otimes_{\mathbb{Z}} i d\right)\right)$ given by

$$
v_{1} \otimes v_{2} \otimes \cdots \otimes v_{n} \mapsto \sum_{\sigma \in \mathfrak{S}_{p}} x_{\sigma}\left(v_{\sigma^{-1}(1)} \otimes \cdots \otimes v_{\sigma^{-1}(n)}\right) .
$$

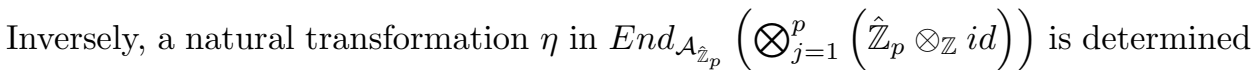
by its value on $\mathbb{Z}^{n}$. Fix a basis $\left\{e_{1}, \ldots, e_{n}\right\}$ of $\mathbb{Z}^{n}$. The image of $e_{1} \otimes e_{2} \otimes \cdots \otimes e_{n}$ under $\eta_{\mathbb{Z}^{n}}$ can be uniquely written as $x_{\eta} \cdot\left(e_{1} \otimes e_{2} \otimes \cdots \otimes e_{n}\right)$ for an $x_{\eta} \in \hat{\mathbb{Z}}_{p} \mathfrak{S}_{n}$. The two mappings $x \mapsto \eta_{x}$ and $\eta \mapsto x_{\eta}$ are mutually inverse and obviously ring homomorphisms. This proves the theorem.

Remark 6.7. The Schur algebra $S_{\hat{\mathbb{Z}}_{p}}(p, p)$ is a classical order which was completely described by König in [20]:

$$
S_{\hat{\mathbb{Z}}_{p}}(p, p)^{\prime} \simeq\left(\begin{array}{cc}
\hat{\mathbb{Z}}_{p} & \hat{\mathbb{Z}}_{p} \\
(p) & \hat{\mathbb{Z}}_{p}
\end{array}\right) \oplus\left(\begin{array}{cc}
\hat{\mathbb{Z}}_{p} & \hat{\mathbb{Z}}_{p} \\
(p) & \hat{\mathbb{Z}}_{p}
\end{array}\right) \oplus \ldots \oplus\left(\begin{array}{cc}
\hat{\mathbb{Z}}_{p} & \hat{\mathbb{Z}}_{p} \\
(p) & \hat{\mathbb{Z}}_{p}
\end{array}\right) \oplus \hat{\mathbb{Z}}_{p},
$$

where we denote by $S_{\hat{\mathbb{Z}}_{p}}(p, p)^{\prime}$ the basic algebra of the Schur algebra $S_{\hat{\mathbb{Z}}_{p}}(p, p)$.

Now, any strict polynomial functor induces a polynomial functor. So, composing further to the Green order lifting the principal block of the group ring of the symmetric group, we get an induced functor

$$
S_{\hat{\mathbb{Z}}_{p}}(p, p)^{\prime}-\bmod \longrightarrow \mathcal{A}_{\hat{\mathbb{Z}}_{p}}^{p} \stackrel{\phi}{\longrightarrow} e \cdot \Lambda \cdot e-\bmod .
$$

Since the functor $\phi$ is induced by the Schur functor, this composed map is induced by the natural embedding of $e \cdot \Lambda \cdot e \hookrightarrow S_{\hat{\mathbb{Z}}_{p}}(p, p)^{\prime}$.

\section{IDENTIFYING THE LATTICES AS FUNCTORS}

We shall identify the indecomposable functors of $\mathcal{A}_{\hat{\mathbb{Z}}_{p}}^{p}$ which correspond to indecomposable $\Gamma_{\hat{\mathbb{Z}}_{p}}$-lattices. We call such polynomial functors 'polynomial lattices'.

The structure of $\Gamma_{\hat{\mathbb{Z}}_{p}, 0}$ implies that there are exactly $3 p-2$ indecomposable $\Gamma_{\hat{\mathbb{Z}}_{p}, 0}$ lattices. Indeed, the indecomposable lattices are the $p$ projective indecomposable modules $P_{1}, P_{2}, \ldots, P_{p}$, the $p-1$ kernels of any fixed non-zero homomorphism $P_{i} \longrightarrow P_{i+1}$ for $i=1,2, \ldots, p-1$, as well as the $p-1$ kernels of any fixed nonzero homomorphism $P_{i} \longrightarrow P_{i-1}$ for $i=2,3, \ldots, p$. Therefore, there are exactly $2 p-2+\rho(p)$ indecomposable 'lattices' of exactly degree $p$ polynomial functors in $\mathcal{A}_{\hat{\mathbb{Z}}_{p}}^{p}$, where $\rho(p)$ denotes the number of partitions of $p$.

Moreover, the proof of Theorem 6.4 shows that exactly the projective indecomposable $\Gamma_{\hat{\mathbb{Z}}_{p}}$-modules of degree $d \in\{2, \ldots, p-1\}$ will give rise to indecomposable lattices. Denote by $\rho(k)$ the number of partitions of $k$ into non-zero integers; we get the following corollary to Theorem 6.4 . 
Corollary 7.1. Up to isomorphism there are exactly $2 p-2+\sum_{k=2}^{p} \rho(k)$ indecomposable polynomial lattices in $\mathcal{A}_{\hat{\mathbb{Z}}_{p}}^{p}$, and $\sum_{k=2}^{p} \rho(k)$ of them are projective, while $2(p-1)$ of them are not projective. The non-projective polynomial lattices are kernels of mappings between projective indecomposable polynomial functors.

\section{ACKNOWLEDGEMENTS}

This research was done during the years 2001 to 2003 as joint work with Steffen König. We presented the result on various occasions, such as in Leicester in March 2003, Jena and Strasbourg in October 2003, in Valenciennes in February 2004, in Bern in February 2005, in Mainz in June 2005, and in October 2005 at the "séminaire Chevalley" Paris. Recently we received much encouragement to publish our manuscript. Steffen König 1 wrote and stated that he would not be able to find the time to finish the paper, and he gave authorization to publish the paper alone. The author wishes to thank Steffen König for having shared his insight with him, and for allowing him to publish the paper.

The author wishes to thank the referee for many helpful comments and in particular for indicating the treatment of Section 3.2 .

\section{REFERENCES}

[1] Maurice Auslander, Idun Reiten, and Sverre O. Smalø, Representation theory of Artin algebras, Cambridge Studies in Advanced Mathematics, vol. 36, Cambridge University Press, Cambridge, 1995. MR1314422 (96c:16015)

[2] Hans Joachim Baues, Quadratic functors and metastable homotopy, J. Pure Appl. Algebra 91 (1994), no. 1-3, 49-107, DOI 10.1016/0022-4049(94)90135-X. MR1255923 (94j:55022)

[3] Hans-Joachim Baues, Winfried Dreckmann, Vincent Franjou, and Teimuraz Pirashvili, Foncteurs polynomiaux et foncteurs de Mackey non linéaires (French, with English and French summaries), Bull. Soc. Math. France 129 (2001), no. 2, 237-257. MR.1871297 (2002j:18004)

[4] D. J. Benson, Representations and cohomology. I. Basic representation theory of finite groups and associative algebras, Cambridge Studies in Advanced Mathematics, vol. 30, Cambridge University Press, Cambridge, 1991. MR.1110581(92m:20005)

[5] Aurélien Djament, Sur l'homologie des groupes unitaires à coefficients polynomiaux (French, with English and French summaries), J. K-Theory 10 (2012), no. 1, 87-139, DOI 10.1017/is012003003jkt184. MR2990563

[6] Aurélien Djament and Christine Vespa, Sur l'homologie des groupes orthogonaux et symplectiques à coefficients tordus (French, with English and French summaries), Ann. Sci. Éc. Norm. Supér. (4) 43 (2010), no. 3, 395-459. MR2667021 (2011h:20095)

[7] Aurélien Djament and Christine Vespa, Sur l'homologie des groupes d'automorphismes des groupes libres à coefficients polynomiaux, preprint 2013, arXiv: 1210.4030v2

[8] Yuriy A. Drozd, Finitely generated quadratic modules, Manuscripta Math. 104 (2001), no. 2, 239-256, DOI 10.1007/s002290170041. MR.1821185(2002d:16021)

[9] Yuriy A. Drozd, On cubic functors, Comm. Algebra 31 (2003), no. 3, 1147-1173, DOI 10.1081/AGB-120017759. MR.1971055 (2004a:16031)

[10] Samuel Eilenberg and Saunders Mac Lane, On the groups $H(\Pi, n)$. III, Ann. of Math. (2) 60 (1954), 513-557. MR0065163 (16,392a)

[11] Vincent Franjou, Extensions entre puissances extérieures et entre puissances symétriques (French), J. Algebra 179 (1996), no. 2, 501-522, DOI 10.1006/jabr.1996.0022. MR.1367860 (96i:20061)

[12] Vincent Franjou, Jean Lannes, and Lionel Schwartz, Autour de la cohomologie de Mac Lane des corps finis (French, with English and French summaries), Invent. Math. 115 (1994), no. 3, 513-538, DOI 10.1007/BF01231771. MR 1262942 (95d:19002)

\footnotetext{
${ }^{1}$ Email to the author from April 12, 2013.
} 
[13] Vincent Franjou and Teimuraz Pirashvili, On the Mac Lane cohomology for the ring of integers, Topology 37 (1998), no. 1, 109-114, DOI 10.1016/S0040-9383(97)00005-0. MR.1480880 (98h:19002)

[14] Vincent Franjou and Teimuraz Pirashvili, Stable K-theory is bifunctor homology (after A. Scorichenko) (English, with English and French summaries), Rational representations, the Steenrod algebra and functor homology, Panor. Synthèses, vol. 16, Soc. Math. France, Paris, 2003, pp. 107-126. MR2117530

[15] Vincent Franjou, Eric M. Friedlander, Alexander Scorichenko, and Andrei Suslin, General linear and functor cohomology over finite fields, Ann. of Math. (2) 150 (1999), no. 2, 663728, DOI 10.2307/121092. MR 1726705 (2001b:14076)

[16] Eric M. Friedlander and Andrei Suslin, Cohomology of finite group schemes over a field, Invent. Math. 127 (1997), no. 2, 209-270, DOI 10.1007/s002220050119. MR 1427618 (98h:14055a)

[17] J. A. Green, Polynomial representations of $\mathrm{GL}_{n}$, Second corrected and augmented edition, Lecture Notes in Mathematics, vol. 830, Springer, Berlin, 2007. With an appendix on Schensted correspondence and Littelmann paths by K. Erdmann, Green and M. Schocker. MR2349209 (2009b:20084)

[18] Manfred Hartl, Teimuraz Pirashvili and Christine Vespa, Polynomial functors from algebras over a set-operad and non-linear Mackey functors, preprint (2012) arxiv:1209.1607v2

[19] Hans-Werner Henn, Jean Lannes, and Lionel Schwartz, The categories of unstable modules and unstable algebras over the Steenrod algebra modulo nilpotent objects, Amer. J. Math. 115 (1993), no. 5, 1053-1106, DOI 10.2307/2375065. MR1246184(94i:55024)

[20] Steffen König, Cyclotomic Schur algebras and blocks of cyclic defect, Canad. Math. Bull. 43 (2000), no. 1, 79-86, DOI 10.4153/CMB-2000-012-0. MR.1749952 (2001f:20022)

[21] Steffen König and Alexander Zimmermann, Derived equivalences for group rings, Lecture Notes in Mathematics, vol. 1685, Springer-Verlag, Berlin, 1998. With contributions by Bernhard Keller, Markus Linckelmann, Jeremy Rickard and Raphaël Rouquier. MR.1649837 (2000g:16018)

[22] Nicholas J. Kuhn, Generic representations of the finite general linear groups and the Steenrod algebra. I, Amer. J. Math. 116 (1994), no. 2, 327-360, DOI 10.2307/2374932. MR.1269607 (95c:55022)

[23] Nicholas J. Kuhn, Generic representations of the finite general linear groups and the Steenrod algebra. II, K-Theory 8 (1994), no. 4, 395-428, DOI 10.1007/BF00961409. MR 1300547 (95k:55038)

[24] Nicholas J. Kuhn, The generic representation theory of finite fields: a survey of basic structure, Infinite length modules (Bielefeld, 1998), Trends Math., Birkhäuser, Basel, 2000, pp. 193-212. MR1789216 (2001m:20065)

[25] Nicholas J. Kuhn, A stratification of generic representation theory and generalized Schur algebras, K-Theory 26 (2002), no. 1, 15-49, DOI 10.1023/A:1016357323204. MR.1918209 (2003h:20089)

[26] Saunders Mac Lane, Categories for the working mathematician, 2nd ed., Graduate Texts in Mathematics, vol. 5, Springer-Verlag, New York, 1998. MR1712872 (2001j:18001)

[27] T. I. Pirashvili, Polynomial functors (Russian, with English summary), Trudy Tbiliss. Mat. Inst. Razmadze Akad. Nauk Gruzin. SSR 91 (1988), 55-66. MR1029007 (91f:18004)

[28] Teimuraz Pirashvili, Polynomial functors over finite fields (after Franjou, Friedlander, Henn, Lannes, Schwartz, Suslin), Astérisque 276 (2002), 369-388. Séminaire Bourbaki, Vol. 1999/2000. MR 1886766 (2003c:20053)

[29] Teimuraz Pirashvili, Introduction to functor homology (English, with English and French summaries), Rational representations, the Steenrod algebra and functor homology, Panor. Synthèses, vol. 16, Soc. Math. France, Paris, 2003, pp. 1-26. MR2117526

[30] Laurent Piriou, Extensions entre foncteurs de la catégorie des espaces vectoriels sur le corps premier à $p$ éléments dans elle-même, thèse de doctorat université Paris 7 (1995).

[31] Laurent Piriou and Lionel Schwartz, Extensions de foncteurs simples (French, with English summary), K-Theory 15 (1998), no. 3, 269-291, DOI 10.1023/A:1007700132156. MR.1659961 (2000g:20085)

[32] Chrysostomos Psaroudakis and Jorge Vitória, Recollements of module categories, Appl. Categ. Structures 22 (2014), no. 4, 579-593, DOI 10.1007/s10485-013-9323-x. MR.3227608 
[33] I. Reiner, Maximal orders, London Mathematical Society Monographs. New Series, vol. 28, The Clarendon Press Oxford University Press, Oxford, 2003. Corrected reprint of the 1975 original. With a foreword by M. J. Taylor. MR1972204 (2004c:16026)

[34] K. W. Roggenkamp, Blocks of cyclic defect and Green-orders, Comm. Algebra 20 (1992), no. 6, 1715-1734, DOI 10.1080/00927879208824426. MR1162603 (93g:20014)

[35] Antoine Touzé, Cohomologie Rationnelle du Groupe Linéaire et Extensions de Bifoncteurs, Thèse de doctorat de l'université de Nantes (2008).

[36] Antoine Touzé and Wilberd van der Kallen, Bifunctor cohomology and cohomological finite generation for reductive groups, Duke Math. J. 151 (2010), no. 2, 251-278, DOI 10.1215/00127094-2009-065. MR2598378 (2011g:20075)

[37] Christine Vespa, Generic representations of orthogonal groups: the mixed functors, Algebr. Geom. Topol. 7 (2007), 379-410, DOI 10.2140/agt.2007.7.379. MR2308951 (2008c:18002)

[38] Christine Vespa, Generic representations of orthogonal groups: the functor category $\mathcal{F}_{\text {quad }}$, J. Pure Appl. Algebra 212 (2008), no. 6, 1472-1499, DOI 10.1016/j.jpaa.2007.10.014. MR:2391661 (2008m:18001)

[39] Christine Vespa, Generic representations of orthogonal groups: projective functors in the category $\mathcal{F}_{\text {quad }}$, Fund. Math. 200 (2008), no. 3, 243-278, DOI 10.4064/fm200-3-2. MR2429598 (2009i:18001)

[40] Lionel Schwartz, Unstable modules over the Steenrod algebra and Sullivan's fixed point set conjecture, Chicago Lectures in Mathematics, University of Chicago Press, Chicago, IL, 1994. MR.1282727 (95d:55017)

Département de Mathématiques et CNRS UMR 7352, Université de Picardie, 33 Rue St Leu, F-80039 Amiens Cedex 1, France

E-mail address: alexander.zimmermann@u-picardie.fr 